\title{
Rigorous plasticity solutions for the bearing capacity of two-layered clays
}

\author{
R. S. MERIFIELD, ${ }^{*}$ S. W. SLOAN* and H. S. YU*
}

This paper applies numerical limit analysis to evaluate the undrained bearing capacity of a rigid surface footing resting on a two-layer clay deposit. Rigorous bounds on the ultimate bearing capacity are obtained by employing finite elements in conjunction with the upper and lower bound limit theorems of classical plasticity. Both methods assume a perfectly plastic soil model with a Tresca yield criterion and generate large linear programming problems. The solution to the lower bound linear programming problem is obtained by modelling a statically admissible stress field, whereas the upper bound problem is solved through modelling a kinematically admissible velocity field. Results from the limit theorems typically bracket the true collapse load to within approximately $12 \%$, and have been presented in the form of bearing capacity factors based on various layer properties and geometries. A comparison is made between existing limit analysis, empirical and semi-empirical solutions. This indicates that the latter can overestimate or underestimate the bearing capacity by as much as $20 \%$ for certain problem geometries.

KEYWORDS: bearing capacity; clays; design; failure; footings/foundations; numerical modelling.
Dans cet exposé, nous servons d'analyses limites numériques pour évaluer la capacité porteuse non drainée d'une assise à surface rigide reposant dur un dépôt argileux à deux couches. On obtient des limites rigoureuses pour la capacité porteuse ultime en employant des éléments finis en conjonction avec les théorèmes de limite à borne supérieure et inférieure de la plasticité classique. Les deux méthodes supposent un modèle de sol parfaitement plastique avec un critère d'élasticité Tresca et entraînent de gros problèmes de programmation linéaire. On obtient la solution au problème de programmation linéaire de limite inférieure en faisant la maquette d'un champ de contrainte admissible en statistique tandis que le problème de limite supérieure est résolu par la mise en maquette d'un champ de vélocité admissible en cinétique. De manière typique, les résultats de théorèmes limites cernent dans une marge d'environ $12 \%$ la vraie charge d'affaissement et sont présentés sous forme de facteurs de capacité porteuse basés sur les propriétés et géométries des diverses couches. Nous comparons les analyses limites existantes, les solutions empiriques et les solutions semiempiriques. Cette comparaison indique que ces dernières peuvent surévaluer ou sous-estimer de $20 \%$ la capacité porteuse dans le cas de certaines géométries à problème.

\section{INTRODUCTION}

The ultimate bearing capacity of surface strip footings resting on a single layer of homogeneous undrained clay has been studied by numerous investigators, with practitioners generally using Terzaghi's (1943) expression to compute ultimate footing loads. In reality, however, soil strength profiles beneath footings are not homogeneous but may increase or decrease with depth or consist of distinct layers having significantly different properties. While the effect of increasing strength with

Manuscript received 18 November 1997; revised manuscript accepted 9 December 1998.

Discussion on this paper closes 5 November; for further details see p. ii.

* University of Newcastle, Australia. depth on bearing capacity has been addressed by several researchers, notably Davis \& Booker (1973), rigorous solutions to the problem of footings resting on layered clays do not appear to exist.

To calculate the ultimate bearing capacity for surface strip footings resting on a horizontally layered clay profile, practitioners commonly use the approximate solutions of Button (1953), Reddy \& Srinivasan (1967), Chen (1975), Brown \& Meyerhof (1969) and Meyerhof \& Hanna (1978). Button (1953) and Chen (1975) calculated upper bound solutions assuming a simple circular failure surface (Fig. 1), while Reddy \& Srinivasan (1967), assuming the same circular mechanism, obtained results using the method of limiting equilibrium. The solutions of Brown \& Meyerhof (1969) and 


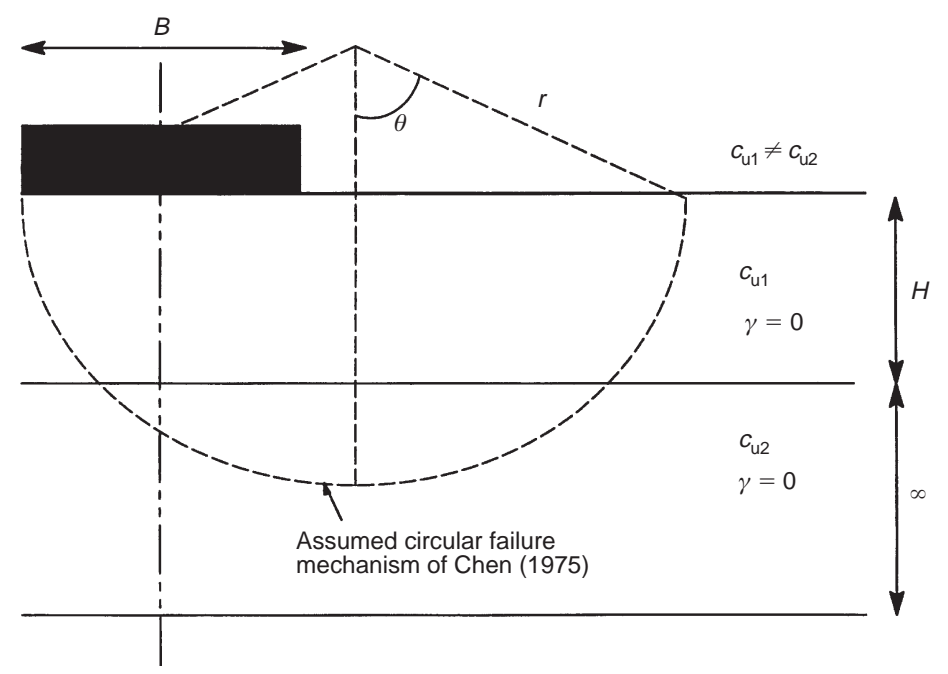

Fig. 1. Problem notation

Meyerhof \& Hanna (1978) were based upon a series of model footing tests from which empirical and semi-empirical solutions for the bearing capacity factor were derived. More recently, an upper bound method of solution was presented by Florkiewicz (1989), who postulated a kinematically admissible failure mechanism consisting of a number of rigid blocks arranged in a Prandtl collapse mode. Although useful, only limited results were produced by Florkiewicz.

Because of its simplicity, the upper bound method has been used widely to estimate the bearing capacity of layered clays. Although an upper bound solution often gives a useful estimate of the bearing capacity, it may lead to a lower factor of safety for design than the real one. A more desirable solution for engineering practice is a lower bound estimate, as it results in a safe design and, if used in conjunction with an upper bound solution, serves to bracket the actual collapse load from above and below.

The purpose of this paper is to take advantage of the ability of the limit theorems to bracket the actual collapse load by computing both types of solution for the bearing capacity of strip footings on a two-layered clay profile. These solutions are obtained using the numerical techniques developed by Sloan (1988) and Sloan \& Kleeman (1995), which are based upon the limit theorems of classical plasticity and finite elements. The methods assume a perfectly plastic soil model with a Tresca yield criterion and lead to large linear programming problems. The solution to the lower bound optimization problem defines a statically admissible stress field and gives a rigorous lower bound on the ultimate bearing capacity. The solution to the upper bound optimization problem defines a kinematically admissible velocity field and hence provides a rigorous upper bound on the ultimate bearing capacity. A statically admissible stress field is one which satisfies equilibrium, the stress boundary conditions and the yield criterion, while a kinematically admissible velocity field is one which satisfies compatibility, the flow rule and the velocity boundary conditions.

An alternative method for predicting the loaddeformation response, and hence collapse, of geotechnical structures is the displacement finite element method. Theoretically, this technique can deal with complicated loadings, excavation and deposition sequences, geometries of arbitrary shape, anisotropy, layered deposits and complex stress-strain relationships. In practice, however, great care must be exercised when finite element analysis is employed to predict limit loads. Even for quite simple problems, experience has indicated that results from the displacement finite element method tend to overestimate the true limit load and, in some instances, fail to provide a clear indication of collapse altogether (Nagtegaal et al., 1974; Sloan \& Randolph, 1982). This phenomenon, which is commonly known as 'locking', restricts the type of element that may be used successfully in limit load computations.

\section{PROBLEM DEFINITION}

The plane strain bearing capacity problem to be considered is illustrated in Fig. 1. A strip footing of width $B$ rests upon an upper layer of clay with 
undrained shear strength $c_{\mathrm{u}}$ and thickness $H$. This is underlain by a clay layer of undrained shear strength $c_{\mathrm{u} 2}$ and infinite depth.

The bearing capacity solution to this problem will be a function of the two ratios $H / B$ and $c_{\mathrm{u} 1} / c_{\mathrm{u} 2}$. Past research by Brown \& Meyerhof (1969) and Meyerhof \& Hanna (1978) indicates that a reduction in bearing capacity for a strongover-soft clay system may occur up to a depth ratio of $H / B \simeq 2.5$. In this paper, solutions have been computed for problems where $H / B$ ranges from 0.125 to 2 and $c_{\mathrm{u} 1} / c_{\mathrm{u} 2}$ varies from 0.2 to 5 . This covers most problems of practical interest. Note that $c_{\mathrm{u} 1} / c_{\mathrm{u} 2}>1$ corresponds to the common case of a strong clay layer over a soft clay layer, while $c_{\mathrm{u} 1} / c_{\mathrm{u} 2}<1$ corresponds to the reverse.

The bearing capacity of a shallow strip footing on a clay layer can be written in the form

$$
q_{\mathrm{u}}=c_{\mathrm{u}} N_{\mathrm{c}}+q
$$

where $N_{\mathrm{c}}$ is a bearing capacity factor and $q$ is a surcharge. For a surface strip footing without a surcharge, this equation reduces to

$$
q_{\mathrm{u}}=c_{\mathrm{u}} N_{\mathrm{c}}
$$

Note that the ultimate bearing capacity for undrained loading of a footing is independent of the soil unit weight. This follows from the fact that the undrained strength is assumed to be independent of the mean normal stress.

For the case of a layered soil profile, it is convenient to rewrite equation (2) in the form

$$
N_{\mathrm{c}}^{*}=\frac{q_{\mathrm{u}}}{c_{\mathrm{u} 1}}
$$

where $c_{\mathrm{u} 1}$ is the undrained shear strength of the top layer, and $N_{\mathrm{c}}^{*}$ is a modified bearing capacity factor which is a function of both $H / B$ and $c_{\mathrm{u} 1} / c_{\mathrm{u} 2}$. The value of $N_{\mathrm{c}}^{*}$ will be computed using the results from both upper and lower bound analyses for each ratio of $H / B$ and $c_{\mathrm{u} 1} / c_{\mathrm{u} 2}$. For a homogeneous profile where $c_{\mathrm{u} 1}=c_{\mathrm{u} 2}, N_{\mathrm{c}}^{*}$ equals the well-known Prantl solution of $(2+\pi)$. For the range of problem geometries considered, the bound solutions are typically able to bracket the exact bearing capacity factor to within $12 \%$ or better.

\section{FINITE ELEMENT FORMULATION OF LIMIT THEOREMS}

The use of special finite element formulations and linear programming to compute lower bound solutions for soil mechanics problems appears to have been first proposed by Lysmer (1970). Similar methods have also been described by Anderheggen \& Knopfel (1972) and Bottero et al. (1980), who also formulated solution procedures for the upper bound limit theorem. Although the procedures presented by these authors were potentially very powerful, they were initially limited by the slowness of the algorithms available to solve the large linear programming problems that were generated. More recently, Sloan (1988) and Sloan \& Kleeman (1995) employed new formulations which used an active set algorithm to solve the large linear programming problems much more efficiently. The speed and modest memory demands of these formulations enable large problems to be solved using a desktop microcomputer.

The following is only a brief summary of the numerical formulation of the limit theorems, and only those aspects specifically related to the current study of bearing capacity are mentioned. Full details of the numerical procedures can be found in Sloan (1988) and Sloan \& Kleeman (1995), and will not be repeated here.

\section{Lower bound formulation}

The lower bound solution is obtained by modelling a statically admissible stress field using finite elements with stress nodal variables, where stress discontinuities can occur at the interface between adjacent elements. Application of the stress boundary conditions, equilibrium equations and yield criterion leads to an expression of the collapse load which is maximized subject to a set of linear constraints on the stresses.

By using finite element methods, the stress field can be modelled under plane strain conditions using the three types of elements shown in Fig. 2. Including extension elements in the lower bound mesh permits the stress field to be extended throughout the semi-infinite domain of the problem without violating equilibrium, the stress boundary conditions, or the yield criterion. The unknown stresses within each element are assumed to vary linearly.

Unlike the more familiar displacement finite element method, each node is unique to a particular element and therefore any number of nodes may share the same coordinates. This enables a wide range of stress fields to be modelled by permitting statically admissible stress discontinuities at all edges that are shared by adjacent elements, including those edges that are shared by adjacent extension elements. To furnish a rigorous lower bound solution for the collapse load, it is necessary to ensure that the stress field obeys equilibrium, the stress boundary conditions and the yield criterion. Each of these requirements imposes a separate set of constraints on the nodal stresses. The present analyses assume that the undrained shear strength of the clay may be represented by the Tresca yield criterion, which is replaced by a series of linear inequalities (see Sloan, 1988). This linear approximation, which is known as a linearized yield surface, is defined to be internal to the 


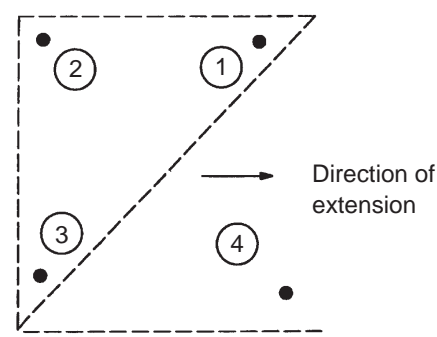

4-noded rectangular extension element

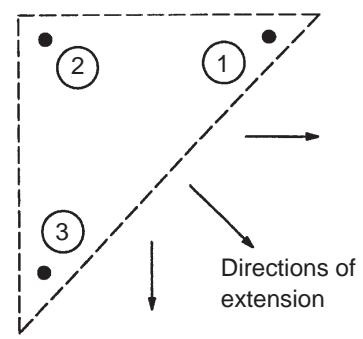

3-noded trianglar extension element
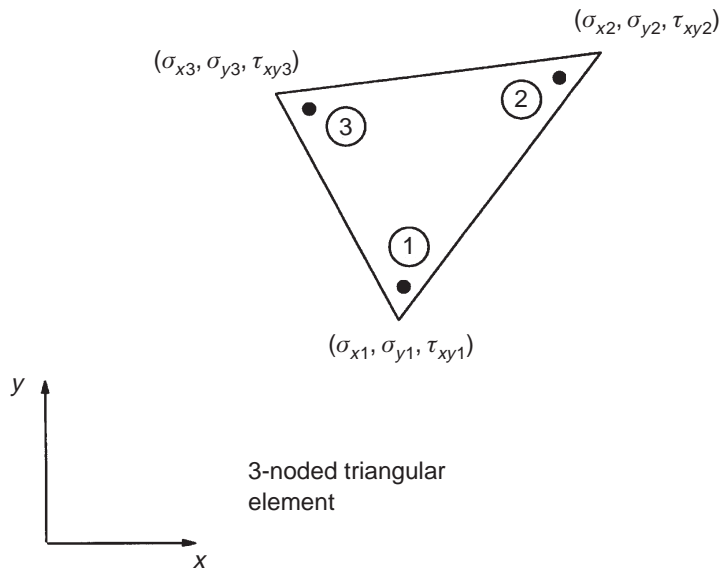

3-noded triangular

element

Fig. 2. Elements for finite element lower bound analysis

Tresca yield surface to preserve the lower bound property of the solution.

For many plane strain geotechnical problems, we seek a statically admissible stress field which maximizes an integral of the normal stress $\sigma_{\mathrm{n}}$ over some part of the boundary. Denoting the out-ofplane thickness by $h$, these integrals are typically of the form

$$
Q=h \int_{S} \sigma_{\mathrm{n}} \mathrm{d} s
$$

where $Q$ represents the collapse load. For the case of equation (4), the integration can be performed analytically, and after substitution of the stress transformation equations, the collapse load $Q$ may be written as

$$
Q=\mathbf{c}^{\mathrm{T}} \mathbf{x}
$$

where $\mathbf{c}^{\mathrm{T}} \mathbf{x}$ is known as the objective function, since it defines the quantity which is to be optimized.

By assembling the various constraints and objective function coefficients for the overall mesh, the problem of finding a statically admissible stress field which maximizes the collapse load may be written as

$$
\begin{array}{ll}
\text { Minimize } & -\mathbf{C}^{\mathrm{T}} \mathbf{X} \\
\text { Subject to } & \mathbf{A}_{1} \mathbf{X}=\mathbf{B}_{1} \\
& \mathbf{A}_{2} \mathbf{X} \leqslant \mathbf{B}_{2}
\end{array}
$$

where $\mathbf{X}$ is the global vector of unknown nodal stresses, $\mathbf{A}_{1}$ is a matrix of constraints generated from equilibrium and stress boundary conditions, and $\mathbf{A}_{2}$ is a matrix of constraints from yield conditions.

A typical lower bound mesh for the problem of a surface footing resting on a layered clay profile, along with the applied stress boundary conditions, is shown in Fig. 3.

To model a perfectly rough footing, no additional constraints are placed on the allowable shear stress at element nodes located directly under the footing. The shear stress is therefore unrestricted and may vary up to the undrained shear strength of the soil (according to the yield constraint). Alter- 


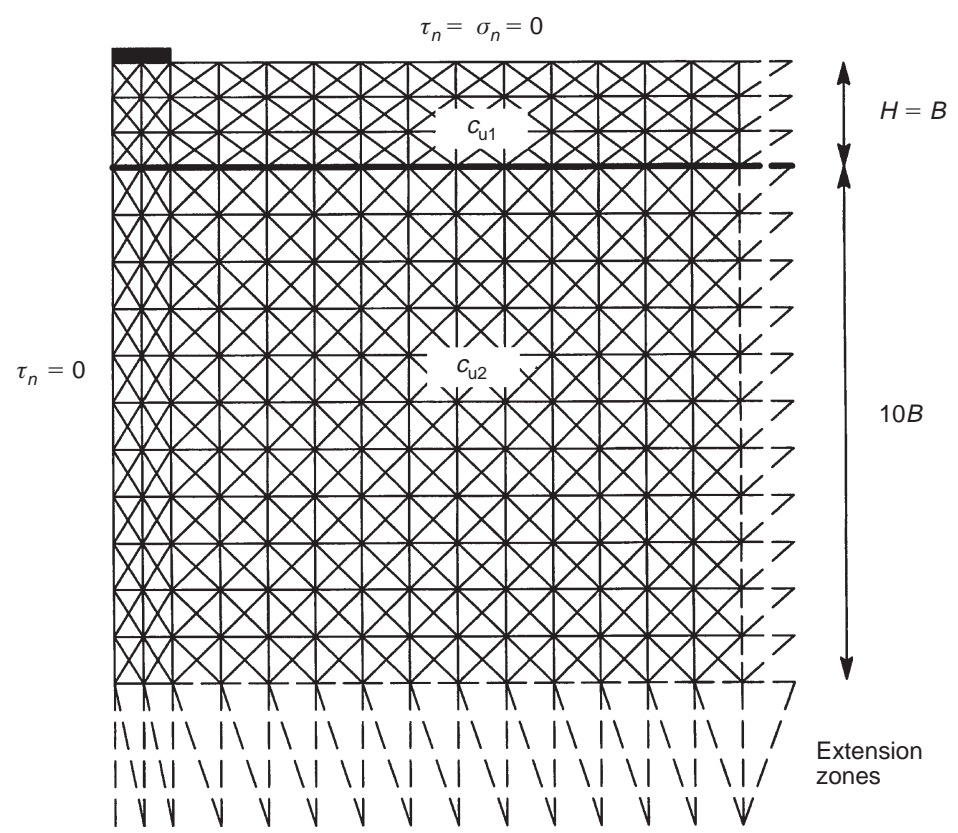

Fig. 3. Typical finite element lower bound mesh $(H / B=1)$

natively, a smooth footing may be modelled by insisting that the shear stress is zero at all element nodes along the footing-soil interface.

A lower bound solution for the footing problem is obtained by maximizing the integral of the compressive stress along the soil-footing interface. The individual normal stresses at element nodes on the soil-footing boundary are unrestricted.

\section{Upper bound formulation}

An upper bound on the exact collapse load can be obtained by modelling a kinematically admissible velocity field. To be kinematically admissible, such a velocity field must satisfy the set of constraints imposed by compatibility, velocity boundary conditions and the flow rule. By prescribing a set of velocities along a specified boundary segment, we can equate the power dissipated internally, due to plastic yielding within the soil mass and sliding of the velocity discontinuities, with the power dissipated by the external loads to yield a strict upper bound on the true limit load. An advantage of using the new upper bound formulation of Sloan \& Kleeman (1995) is that the direction of shearing of each velocity discontinuity is found automatically and need not be specified $a$ priori. A good indication of the failure mechanism can therefore be obtained without any assumptions being made in advance.
As in the lower bound case, a linear approximation to the Tresca yield surface is adopted to ensure that the formulation results in a linear programming problem. Unlike the lower bound formulation, however, this surface must be external to the parent yield surface to ensure that the solution found is a rigorous upper bound on the exact collapse load. This is achieved by adopting a $p$-sided prism that circumscribes the Tresca yield surface.

The three-noded triangle is again used for the upper bound formulation. Now, however, each node is associated with two unknown velocities and each element has $p$ non-negative plastic multiplier rates (where $p$ is the number of sides in the linearized yield criterion) as shown in Fig. 4. A linear variation of the velocities is assumed within each triangle. For each velocity discontinuity, there are also four non-negative discontinuity parameters that describe the velocity jumps along each triangle edge (see Sloan \& Kleeman, 1995).

To define the objective function, the dissipated power (or some related load parameter) is expressed in terms of the unknown plastic multiplier rates and discontinuity parameters. As the soil deforms, power dissipation may occur in the velocity discontinuities as well as in the triangles.

Once the constraints and the objective function coefficients are assembled, the task of finding a kinematically admissible velocity field, which 


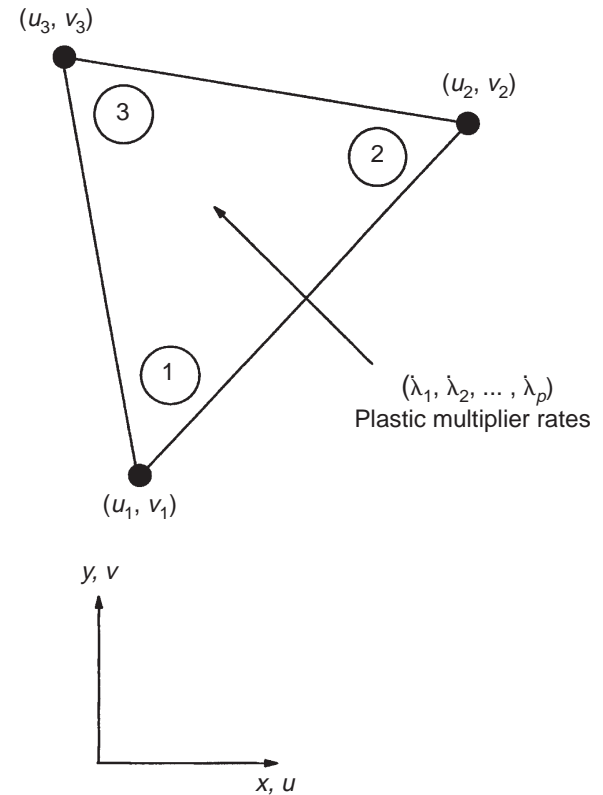

Fig. 4. Element for finite element upper bound analysis minimizes the internal power dissipation for a specified set of boundary conditions, may be written as

$$
\begin{array}{ll}
\text { Minimize } & \mathbf{C}_{2}^{\mathrm{T}} \mathbf{X}_{2}+\mathbf{C}_{3}^{\mathrm{T}} \mathbf{X}_{3} \\
\text { Subject to } & \mathbf{A}_{11} \mathbf{X}_{1}+\mathbf{A}_{12} \mathbf{X}_{2}=0 \\
& \mathbf{A}_{21} \mathbf{X}_{1}+\mathbf{A}_{23} \mathbf{X}_{3}=0 \\
& \mathbf{A}_{31} \mathbf{X}_{1}=\mathbf{B}_{3} \\
& \mathbf{A}_{41} \mathbf{X}_{1}=\mathbf{B}_{4} \\
& \mathbf{X}_{2} \geqslant 0 \\
& \mathbf{X}_{3} \geqslant 0
\end{array}
$$

where $\mathbf{X}_{1}$ is a global vector of nodal velocities, $\mathbf{X}_{2}$ is a global vector of plastic multiplier rates, and $\mathbf{X}_{3}$ is a global vector of discontinuity parameters. The matrices $\mathbf{A}_{11}, \mathbf{A}_{12}$ and $\mathbf{A}_{21}, \mathbf{A}_{23}$ are matrices generated from constraints on plastic flow in the continuum and plastic shearing in velocity discontinuities respectively, and $\mathbf{A}_{31}, \mathbf{A}_{41}$ are matrices generated from constraints due to the velocity boundary conditions.

A typical upper bound mesh for the problem of a surface footing resting on a layered clay profile, along with the applied velocity boundary conditions, is shown in Fig. 5. A line element has been added, enabling various types of footing problems
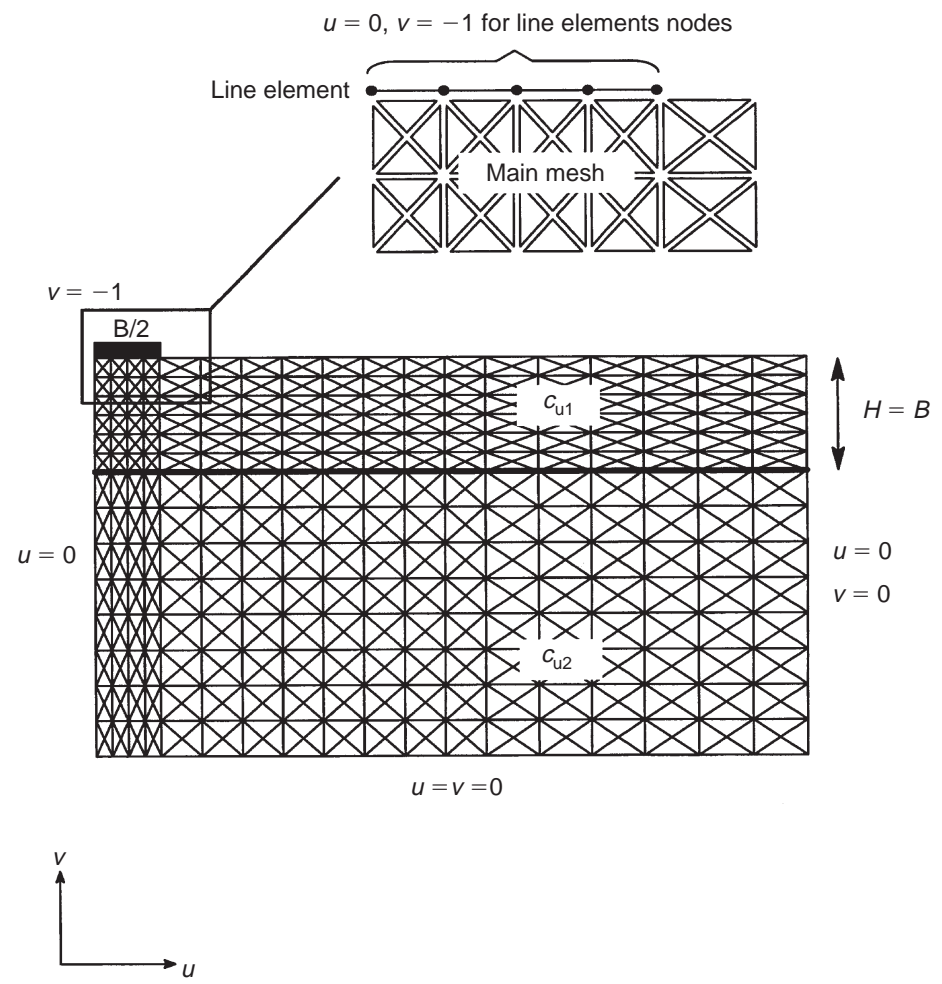

Fig. 5. Typical finite element upper bound mesh $\left(H / B=1, c_{\mathrm{u} 1} / c_{\mathrm{u} 2}=2\right)$ 
to be analysed (i.e. rough, smooth, rigid, flexible). The addition of this line element creates a series of velocity discontinuities between the footing base and the soil which can then be assigned suitable material properties to solve the problem at hand. For example, these velocity discontinuities are assigned a strength equal to the undrained shear strength of the soil for the case of a perfectly rough footing, and a strength of zero for the case of a perfectly smooth footing.

An upper bound solution is obtained by prescribing a unit downward velocity to the nodes along the line element, with the additional constraint that it cannot move horizontally $(u=0)$. After the corresponding optimization problem is solved for the imposed boundary conditions, the collapse load is found by equating the internally dissipated power to the power expended by the external forces. The results for the simple case of a surface footing resting on a homogeneous soil profile are shown in Fig. 6, where the bearing capacity factor $N_{\mathrm{c}}^{*}$ was found to equal 5.32 (approximately 3\% above the exact Prandtl solution of $N_{\mathrm{c}}^{*}=2+\pi$ ).

\section{RESULTS AND DISCUSSION}

The computed upper and lower bound estimates of the bearing capacity factor $N_{\mathrm{c}}^{*}$ for layered clay soils are given in Tables 1 and 2 and shown graphically in Figs 7-10. These results indicate that, for practical design purposes, sufficiently small error bounds were achieved, with the true collapse load typically being bracketed to within $12 \%$ or better.

Figures 7-10 also compare the numerical bounds and the available upper bound solutions of Chen (1975), the empirical solutions obtained by Brown \& Meyerhof (1969), and the semi-empirical solutions of Meyerhof \& Hanna (1978).

The bearing capacity factors obtained by Chen (1975) were obtained by assuming a circular failure mechanism as shown in Fig. 1. By equating the rate of internal and external work, an upper bound expression for the bearing capacity factor is given by

$$
N_{\mathrm{c}}^{*}(r, \theta)=2\left(\frac{r}{B}\right)^{2}\left\{\frac{\theta+n \theta_{i}}{(r / B) \sin \theta-1 / 2}\right\}
$$
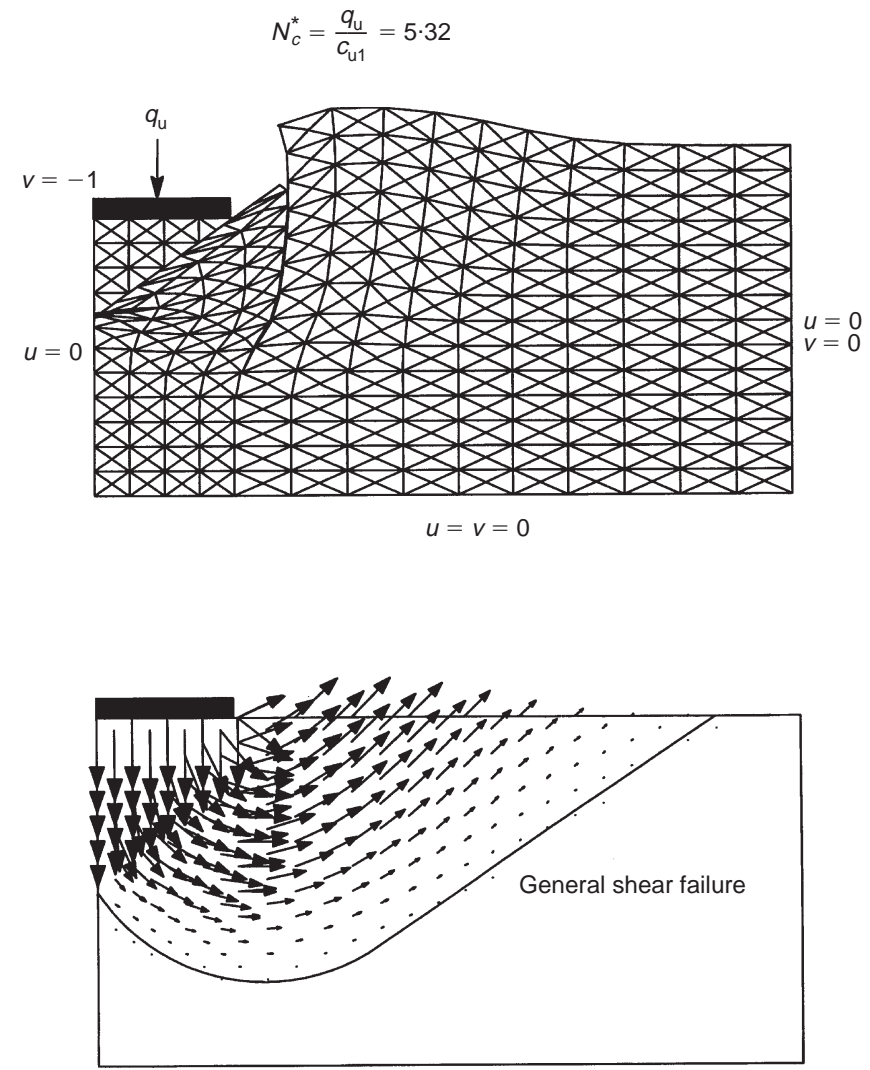

Fig. 6. Deflected mesh and velocity diagram for a homogeneous soil profile 
Table 1. Values of bearing capacity factor $N_{\mathrm{c}}^{*}$ for $c_{\mathrm{u} 1} / c_{\mathrm{u} 2}>1$

\begin{tabular}{|c|c|c|c|c|c|c|}
\hline \multirow[t]{2}{*}{$H / B$} & \multirow[t]{2}{*}{$c_{\mathrm{u} 1 / c_{\mathrm{u} 2}}$} & \multicolumn{5}{|c|}{ Values of bearing capacity factor $N_{\mathrm{c}}^{*}$} \\
\hline & & $\begin{array}{l}\text { Lower bound } \\
\text { (a) }\end{array}$ & $\begin{array}{l}\text { Upper bound } \\
\text { (b) }\end{array}$ & $\begin{array}{c}\text { Average } \\
((\mathrm{a})+(\mathrm{b})) / 2\end{array}$ & $\begin{array}{l}\text { Upper bound } \\
\text { (Chen, 1975) }\end{array}$ & $\begin{array}{c}\text { Meyerhof \& } \\
\text { Hanna (1978) }\end{array}$ \\
\hline $0 \cdot 125$ & $\begin{array}{l}5 \\
4 \\
3 \cdot 5 \\
3 \\
2 \cdot 5 \\
2 \\
1 \cdot 75 \\
1 \cdot 5 \\
1 \cdot 25 \\
\end{array}$ & $\begin{array}{l}1 \cdot 30 \\
1 \cdot 56 \\
1 \cdot 74 \\
1.97 \\
2 \cdot 28 \\
2 \cdot 73 \\
3 \cdot 05 \\
3 \cdot 45 \\
4 \cdot 01\end{array}$ & $\begin{array}{l}1 \cdot 55 \\
1 \cdot 82 \\
2 \cdot 01 \\
2 \cdot 27 \\
2 \cdot 61 \\
3 \cdot 09 \\
3 \cdot 47 \\
3 \cdot 93 \\
4 \cdot 52\end{array}$ & $\begin{array}{l}1 \cdot 43 \\
1 \cdot 69 \\
1 \cdot 88 \\
2 \cdot 12 \\
2 \cdot 44 \\
2 \cdot 91 \\
3 \cdot 26 \\
3 \cdot 69 \\
4 \cdot 27\end{array}$ & $\begin{array}{l}1 \cdot 53 \\
1 \cdot 79 \\
1 \cdot 97 \\
2 \cdot 21 \\
2 \cdot 55 \\
3 \cdot 05 \\
3 \cdot 41 \\
3 \cdot 88 \\
4 \cdot 54\end{array}$ & $\begin{array}{l}1 \cdot 23 \\
1 \cdot 49 \\
1 \cdot 68 \\
1 \cdot 93 \\
2 \cdot 28 \\
2 \cdot 81 \\
3 \cdot 18 \\
3 \cdot 67 \\
4 \cdot 36\end{array}$ \\
\hline $0 \cdot 25$ & $\begin{array}{l}5 \\
4 \\
3 \cdot 5 \\
3 \\
2 \cdot 5 \\
2 \\
1 \cdot 75 \\
1 \cdot 5 \\
1 \cdot 25\end{array}$ & $\begin{array}{l}1 \cdot 60 \\
1 \cdot 87 \\
2 \cdot 05 \\
2 \cdot 27 \\
2 \cdot 58 \\
3 \cdot 01 \\
3 \cdot 29 \\
3 \cdot 65 \\
4 \cdot 10\end{array}$ & $\begin{array}{l}1 \cdot 85 \\
2 \cdot 12 \\
2 \cdot 31 \\
2 \cdot 56 \\
2 \cdot 88 \\
3 \cdot 34 \\
3 \cdot 67 \\
4 \cdot 08 \\
4 \cdot 60\end{array}$ & $\begin{array}{l}1 \cdot 73 \\
1 \cdot 99 \\
2 \cdot 18 \\
2 \cdot 42 \\
2 \cdot 73 \\
3 \cdot 17 \\
3 \cdot 48 \\
3 \cdot 87 \\
4 \cdot 35\end{array}$ & $\begin{array}{l}1 \cdot 82 \\
2 \cdot 12 \\
2 \cdot 32 \\
2 \cdot 58 \\
2 \cdot 92 \\
3 \cdot 40 \\
3 \cdot 73 \\
4 \cdot 14 \\
4 \cdot 69\end{array}$ & $\begin{array}{l}1 \cdot 42 \\
1 \cdot 70 \\
1 \cdot 89 \\
2 \cdot 15 \\
3 \cdot 04 \\
3 \cdot 42 \\
3 \cdot 92 \\
4 \cdot 61 \\
5 \cdot 14\end{array}$ \\
\hline $0 \cdot 375$ & $\begin{array}{l}5 \\
4 \\
3 \cdot 5 \\
3 \\
2 \cdot 5 \\
2 \\
1 \cdot 75 \\
1 \cdot 5 \\
1 \cdot 25\end{array}$ & $\begin{array}{l}1 \cdot 89 \\
2 \cdot 16 \\
2 \cdot 34 \\
2 \cdot 57 \\
2 \cdot 81 \\
3 \cdot 27 \\
3 \cdot 54 \\
3 \cdot 87 \\
4 \cdot 27\end{array}$ & $\begin{array}{l}2 \cdot 13 \\
2 \cdot 42 \\
2 \cdot 61 \\
2 \cdot 90 \\
3 \cdot 20 \\
3 \cdot 65 \\
3 \cdot 93 \\
4 \cdot 28 \\
4 \cdot 78\end{array}$ & $\begin{array}{l}2 \cdot 01 \\
2 \cdot 29 \\
2 \cdot 47 \\
2 \cdot 73 \\
3 \cdot 01 \\
3 \cdot 46 \\
3 \cdot 74 \\
4 \cdot 08 \\
4 \cdot 53\end{array}$ & $\begin{array}{l}2 \cdot 25 \\
2 \cdot 50 \\
2 \cdot 67 \\
2 \cdot 89 \\
3 \cdot 19 \\
3 \cdot 62 \\
3 \cdot 91 \\
4 \cdot 29 \\
5 \cdot 53\end{array}$ & $\begin{array}{l}1 \cdot 62 \\
1 \cdot 91 \\
2 \cdot 11 \\
2 \cdot 37 \\
2 \cdot 74 \\
3 \cdot 28 \\
3 \cdot 66 \\
4 \cdot 16 \\
4 \cdot 86\end{array}$ \\
\hline $0 \cdot 5$ & $\begin{array}{l}5 \\
4 \\
3 \cdot 5 \\
3 \\
2 \cdot 5 \\
2 \\
1 \cdot 75 \\
1 \cdot 5 \\
1 \cdot 25\end{array}$ & $\begin{array}{l}2 \cdot 16 \\
2 \cdot 44 \\
2 \cdot 62 \\
2 \cdot 84 \\
3 \cdot 13 \\
3 \cdot 52 \\
3 \cdot 77 \\
4 \cdot 07 \\
4 \cdot 42\end{array}$ & $\begin{array}{l}2 \cdot 44 \\
2 \cdot 74 \\
2 \cdot 93 \\
3 \cdot 16 \\
3 \cdot 47 \\
3 \cdot 89 \\
4 \cdot 16 \\
4 \cdot 48 \\
4 \cdot 94\end{array}$ & $\begin{array}{l}2 \cdot 30 \\
2 \cdot 59 \\
2 \cdot 77 \\
3 \cdot 00 \\
3 \cdot 30 \\
3 \cdot 70 \\
3 \cdot 97 \\
4 \cdot 28 \\
4 \cdot 68\end{array}$ & $\begin{array}{l}2 \cdot 55 \\
2 \cdot 83 \\
3 \cdot 02 \\
3 \cdot 25 \\
3 \cdot 54 \\
3 \cdot 94 \\
4 \cdot 20 \\
4 \cdot 52 \\
4 \cdot 93\end{array}$ & $\begin{array}{l}1 \cdot 82 \\
2 \cdot 11 \\
2 \cdot 32 \\
2 \cdot 59 \\
2 \cdot 97 \\
3 \cdot 51 \\
3 \cdot 90 \\
4 \cdot 41 \\
5 \cdot 10\end{array}$ \\
\hline $0 \cdot 75$ & $\begin{array}{l}5 \\
4 \\
3 \cdot 5 \\
3 \\
2 \cdot 5 \\
2 \\
1 \cdot 75 \\
1 \cdot 5 \\
1 \cdot 25\end{array}$ & $\begin{array}{l}2 \cdot 64 \\
2 \cdot 96 \\
3 \cdot 14 \\
3 \cdot 36 \\
3 \cdot 64 \\
4 \cdot 00 \\
4 \cdot 21 \\
4 \cdot 44 \\
4 \cdot 70\end{array}$ & $\begin{array}{l}2 \cdot 98 \\
3 \cdot 28 \\
3 \cdot 48 \\
3 \cdot 72 \\
4 \cdot 01 \\
4 \cdot 37 \\
4 \cdot 66 \\
4 \cdot 94 \\
5 \cdot 20\end{array}$ & $\begin{array}{l}2 \cdot 81 \\
3 \cdot 12 \\
3 \cdot 31 \\
3 \cdot 54 \\
3 \cdot 83 \\
4 \cdot 18 \\
4 \cdot 43 \\
4 \cdot 69 \\
4 \cdot 95\end{array}$ & $\begin{array}{l}3 \cdot 28 \\
3 \cdot 53 \\
3 \cdot 69 \\
3 \cdot 88 \\
4 \cdot 12 \\
4 \cdot 43 \\
4 \cdot 63 \\
4 \cdot 87 \\
5 \cdot 17\end{array}$ & $\begin{array}{l}2 \cdot 22 \\
2 \cdot 53 \\
2 \cdot 75 \\
3 \cdot 03 \\
3 \cdot 42 \\
3 \cdot 99 \\
4 \cdot 38 \\
4 \cdot 90 \\
5 \cdot 14\end{array}$ \\
\hline 1 & $\begin{array}{l}5 \\
4 \\
3 \cdot 5 \\
3 \\
2 \cdot 5 \\
2 \\
1 \cdot 75 \\
1 \cdot 5 \\
1 \cdot 25\end{array}$ & $\begin{array}{l}3 \cdot 10 \\
3 \cdot 46 \\
3 \cdot 69 \\
3 \cdot 89 \\
4 \cdot 14 \\
4 \cdot 44 \\
4 \cdot 60 \\
4 \cdot 77 \\
4 \cdot 87\end{array}$ & $\begin{array}{l}3 \cdot 54 \\
3 \cdot 83 \\
4 \cdot 02 \\
4 \cdot 24 \\
4 \cdot 50 \\
4 \cdot 82 \\
5 \cdot 00 \\
5 \cdot 18 \\
5 \cdot 30\end{array}$ & $\begin{array}{l}3 \cdot 32 \\
3 \cdot 65 \\
3 \cdot 85 \\
4 \cdot 07 \\
4 \cdot 32 \\
4 \cdot 63 \\
4 \cdot 80 \\
4 \cdot 97 \\
5 \cdot 09\end{array}$ & $\begin{array}{l}3 \cdot 87 \\
4 \cdot 14 \\
4 \cdot 31 \\
4 \cdot 52 \\
4 \cdot 77 \\
5 \cdot 11 \\
5 \cdot 32 \\
5 \cdot 53 \\
5 \cdot 53\end{array}$ & $\begin{array}{l}2 \cdot 62 \\
2 \cdot 94 \\
3 \cdot 17 \\
3 \cdot 47 \\
3 \cdot 87 \\
4 \cdot 46 \\
4 \cdot 86 \\
5 \cdot 14 \\
5 \cdot 14\end{array}$ \\
\hline
\end{tabular}


Table 1. Continued

\begin{tabular}{|c|c|c|c|c|c|c|}
\hline \multirow[t]{2}{*}{$H / B$} & \multirow[t]{2}{*}{$c_{\mathrm{u} 1} / c_{\mathrm{u} 2}$} & \multicolumn{5}{|c|}{ Values of bearing capacity factor $N_{\mathrm{c}}^{*}$} \\
\hline & & $\begin{array}{l}\text { Lower bound } \\
\text { (a) }\end{array}$ & $\begin{array}{l}\text { Upper bound } \\
\text { (b) }\end{array}$ & $\begin{array}{c}\text { Average } \\
((\mathrm{a})+(\mathrm{b})) / 2\end{array}$ & $\begin{array}{l}\text { Upper bound } \\
\text { (Chen, 1975) }\end{array}$ & $\begin{array}{c}\text { Meyerhof \& } \\
\text { Hanna (1978) }\end{array}$ \\
\hline 1.5 & $\begin{array}{l}5 \\
4 \\
3 \cdot 5 \\
3 \\
2 \cdot 5 \\
2 \\
1 \cdot 75 \\
1 \cdot 5 \\
1 \cdot 25\end{array}$ & $\begin{array}{l}3 \cdot 89 \\
4 \cdot 24 \\
4 \cdot 46 \\
4 \cdot 69 \\
4 \cdot 84 \\
4 \cdot 87 \\
4 \cdot 87 \\
4 \cdot 87 \\
4 \cdot 87\end{array}$ & $\begin{array}{l}4 \cdot 56 \\
4 \cdot 84 \\
4 \cdot 98 \\
5 \cdot 15 \\
5 \cdot 32 \\
\mathbf{5} \cdot 31 \\
\mathbf{5} \cdot 31 \\
\mathbf{5 \cdot 3 1} \\
\mathbf{5 \cdot 2 7}\end{array}$ & $\begin{array}{l}4 \cdot 23 \\
4 \cdot 54 \\
4 \cdot 72 \\
4 \cdot 92 \\
5 \cdot 08 \\
5 \cdot 09 \\
5 \cdot 09 \\
5 \cdot 09 \\
5 \cdot 07\end{array}$ & $\begin{array}{l}5 \cdot 18 \\
5 \cdot 46 \\
\mathbf{5 \cdot 5 3} \\
\mathbf{5 \cdot 5 3} \\
\mathbf{5 \cdot 5 3} \\
\mathbf{5 \cdot 5 3} \\
\mathbf{5 \cdot 5 3} \\
\mathbf{5 \cdot 5 3} \\
\mathbf{5 \cdot 5 3}\end{array}$ & $\begin{array}{l}3 \cdot 41 \\
3 \cdot 77 \\
4 \cdot 02 \\
4 \cdot 35 \\
4 \cdot 78 \\
5 \cdot 14 \\
5 \cdot 14 \\
5 \cdot 14 \\
5 \cdot 14\end{array}$ \\
\hline 2 & $\begin{array}{l}5 \\
4 \\
3 \cdot 5 \\
3 \\
2 \cdot 5 \\
2 \\
1 \cdot 75 \\
1 \cdot 5 \\
1 \cdot 25\end{array}$ & $\begin{array}{l}4 \cdot 61 \\
4 \cdot 81 \\
4 \cdot 81 \\
4 \cdot 81 \\
4 \cdot 81 \\
4 \cdot 81 \\
4 \cdot 81 \\
4 \cdot 81 \\
4 \cdot 81\end{array}$ & $\begin{array}{l}\mathbf{5 \cdot 3 2} \\
\mathbf{5 \cdot 3 2} \\
\mathbf{5 \cdot 3 2} \\
\mathbf{5} \cdot 27 \\
\mathbf{5 \cdot 2 7} \\
\mathbf{5 \cdot 2 7} \\
\mathbf{5 \cdot 2 6} \\
\mathbf{5 \cdot 2 6} \\
\mathbf{5 \cdot 2 6}\end{array}$ & $\begin{array}{l}4 \cdot 96 \\
5 \cdot 06 \\
5 \cdot 06 \\
5 \cdot 04 \\
5 \cdot 04 \\
5 \cdot 04 \\
5 \cdot 04 \\
5 \cdot 04 \\
5 \cdot 04\end{array}$ & $\begin{array}{l}\mathbf{5 \cdot 5 3} \\
\mathbf{5 \cdot 5 3} \\
\mathbf{5 \cdot 5 3} \\
\mathbf{5 \cdot 5 3} \\
\mathbf{5 \cdot 5 3} \\
\mathbf{5 \cdot 5 3} \\
\mathbf{5 \cdot 5 3} \\
\mathbf{5 \cdot 5 3} \\
\mathbf{5 \cdot 5 3}\end{array}$ & $\begin{array}{l}4 \cdot 20 \\
4 \cdot 60 \\
4 \cdot 87 \\
5 \cdot 14 \\
5 \cdot 14 \\
5 \cdot 14 \\
5 \cdot 14 \\
5 \cdot 14 \\
5 \cdot 14\end{array}$ \\
\hline
\end{tabular}

Bold signifies cases where the zone of plastic yielding does not penetrate the bottom layer. The exact solution will be $N_{\mathrm{c}}^{*}=5 \cdot 14$, the Prandtl solution.

Table 2. Values of bearing capacity factor $N_{\mathrm{c}}^{*}$ for $c_{\mathrm{u} 1} / c_{\mathrm{u} 2} \leqslant 1$

\begin{tabular}{|c|c|c|c|c|c|c|}
\hline \multirow[t]{2}{*}{$H / B$} & \multirow[t]{2}{*}{$c_{\mathrm{u} 1} / c_{\mathrm{u} 2}$} & \multicolumn{5}{|c|}{ Values of bearing capacity factor $N_{\mathrm{c}}^{*}$} \\
\hline & & $\begin{array}{l}\text { Lower bound } \\
\text { (a) }\end{array}$ & $\begin{array}{l}\text { Upper bound } \\
\text { (b) }\end{array}$ & $\begin{array}{c}\text { Average } \\
((\mathrm{a})+(\mathrm{b})) / 2\end{array}$ & $\begin{array}{l}\text { Upper bound } \\
\text { (Chen, 1975) }\end{array}$ & $\begin{array}{c}\text { Brown \& } \\
\text { Meyerhof (1969) }\end{array}$ \\
\hline $0 \cdot 125$ & $\begin{array}{l}1 \\
0 \cdot 8 \\
0 \cdot 66 \\
0 \cdot 57 \\
0 \cdot 5 \\
0 \cdot 4 \\
0 \cdot 33 \\
0 \cdot 25 \\
0 \cdot 2\end{array}$ & $\begin{array}{l}4 \cdot 94 \\
5 \cdot 87 \\
6 \cdot 71 \\
7 \cdot 21 \\
7 \cdot 78 \\
7 \cdot 78 \\
7 \cdot 78 \\
7 \cdot 78 \\
7 \cdot 78\end{array}$ & $\begin{array}{l}5 \cdot 32 \\
6 \cdot 36 \\
7 \cdot 27 \\
8 \cdot 03 \\
8 \cdot 55 \\
8 \cdot 55 \\
8 \cdot 55 \\
8 \cdot 55 \\
8 \cdot 55\end{array}$ & $\begin{array}{l}5 \cdot 13 \\
6 \cdot 11 \\
6 \cdot 99 \\
7 \cdot 62 \\
8 \cdot 16 \\
8 \cdot 17 \\
8 \cdot 17 \\
8 \cdot 17 \\
8 \cdot 17\end{array}$ & $\begin{array}{r}5 \cdot 53 \\
7 \cdot 48 \\
8 \cdot 78 \\
9 \cdot 70 \\
10 \cdot 40 \\
10 \cdot 40 \\
10 \cdot 40 \\
10 \cdot 40 \\
10 \cdot 40\end{array}$ & $\begin{array}{c}5 \cdot 14 \\
5 \cdot 81 \\
6 \cdot 38 \\
6 \cdot 71 \\
6 \cdot 91 \\
- \\
- \\
- \\
-\end{array}$ \\
\hline $0 \cdot 25$ & $\begin{array}{l}1 \\
0 \cdot 8 \\
0.66 \\
0 \cdot 57 \\
0 \cdot 5 \\
0 \cdot 4 \\
0 \cdot 33 \\
0 \cdot 25 \\
0 \cdot 2\end{array}$ & $\begin{array}{l}4 \cdot 94 \\
5 \cdot 51 \\
5 \cdot 99 \\
5 \cdot 99 \\
5 \cdot 99 \\
5 \cdot 99 \\
5 \cdot 99 \\
5.99 \\
5 \cdot 99\end{array}$ & $\begin{array}{l}5 \cdot 32 \\
6 \cdot 25 \\
6 \cdot 52 \\
6 \cdot 52 \\
6 \cdot 52 \\
6 \cdot 52 \\
6 \cdot 52 \\
6 \cdot 52 \\
6 \cdot 52\end{array}$ & $\begin{array}{l}5 \cdot 13 \\
5 \cdot 88 \\
5 \cdot 98 \\
6 \cdot 26 \\
6 \cdot 26 \\
6 \cdot 26 \\
6 \cdot 26 \\
6 \cdot 26 \\
6 \cdot 26\end{array}$ & $\begin{array}{l}5 \cdot 53 \\
6 \cdot 57 \\
7 \cdot 61 \\
7 \cdot 61 \\
7 \cdot 61 \\
7 \cdot 61 \\
7 \cdot 61 \\
7 \cdot 61 \\
7 \cdot 61\end{array}$ & $\begin{array}{c}5 \cdot 14 \\
5 \cdot 52 \\
5 \cdot 81 \\
5 \cdot 91 \\
6 \cdot 00 \\
- \\
- \\
- \\
-\end{array}$ \\
\hline $0 \cdot 375$ & $\begin{array}{l}1 \\
0 \cdot 8 \\
0 \cdot 66 \\
0 \cdot 57 \\
0 \cdot 5 \\
0 \cdot 4 \\
0 \cdot 33 \\
0 \cdot 25 \\
0 \cdot 2\end{array}$ & $\begin{array}{l}4 \cdot 94 \\
5 \cdot 38 \\
5 \cdot 40 \\
5 \cdot 40 \\
5 \cdot 40 \\
5 \cdot 40 \\
5 \cdot 40 \\
5 \cdot 40 \\
5 \cdot 40\end{array}$ & $\begin{array}{l}5 \cdot 32 \\
5 \cdot 84 \\
5 \cdot 84 \\
5 \cdot 84 \\
5 \cdot 84 \\
5 \cdot 84 \\
5 \cdot 84 \\
5 \cdot 84 \\
5 \cdot 84\end{array}$ & $\begin{array}{l}5 \cdot 13 \\
5 \cdot 61 \\
5 \cdot 62 \\
5 \cdot 62 \\
5 \cdot 62 \\
5 \cdot 62 \\
5 \cdot 62 \\
5 \cdot 62 \\
5 \cdot 62\end{array}$ & $\begin{array}{l}5 \cdot 53 \\
6 \cdot 24 \\
6 \cdot 24 \\
6 \cdot 24 \\
6 \cdot 24 \\
6 \cdot 24 \\
6 \cdot 24 \\
6 \cdot 24 \\
6 \cdot 24\end{array}$ & $\begin{array}{c}5 \cdot 14 \\
5 \cdot 25 \\
5 \cdot 38 \\
5 \cdot 43 \\
5 \cdot 48 \\
- \\
- \\
- \\
-\end{array}$ \\
\hline
\end{tabular}


Table 2. Continued

\begin{tabular}{|c|c|c|c|c|c|c|}
\hline \multirow[t]{2}{*}{$H / B$} & \multirow[t]{2}{*}{$c_{\mathrm{u} 1} / c_{\mathrm{u} 2}$} & \multicolumn{5}{|c|}{ Values of bearing capacity factor $N_{\mathrm{c}}^{*}$} \\
\hline & & $\begin{array}{l}\text { Lower bound } \\
\text { (a) }\end{array}$ & $\begin{array}{l}\text { Upper bound } \\
\text { (b) }\end{array}$ & $\begin{array}{c}\text { Average } \\
((\mathrm{a})+(\mathrm{b})) / 2\end{array}$ & $\begin{array}{l}\text { Upper bound } \\
\text { (Chen, 1975) }\end{array}$ & $\begin{array}{c}\text { Brown \& } \\
\text { Meyerhof (1969) }\end{array}$ \\
\hline $0 \cdot 5$ & $\begin{array}{l}1 \\
0 \cdot 8 \\
0 \cdot 66 \\
0 \cdot 57 \\
0 \cdot 5 \\
0 \cdot 4 \\
0 \cdot 33 \\
0 \cdot 25 \\
0 \cdot 2\end{array}$ & $\begin{array}{l}4.94 \\
4.98 \\
4.98 \\
4.98 \\
4.98 \\
4.98 \\
4.98 \\
4.98 \\
4.98\end{array}$ & $\begin{array}{l}5 \cdot 32 \\
5 \cdot 49 \\
5 \cdot 49 \\
5 \cdot 49 \\
5 \cdot 49 \\
5 \cdot 49 \\
5 \cdot 49 \\
5 \cdot 49 \\
5 \cdot 49\end{array}$ & $\begin{array}{l}5 \cdot 13 \\
5 \cdot 24 \\
5 \cdot 24 \\
5 \cdot 24 \\
5 \cdot 24 \\
5 \cdot 24 \\
5 \cdot 24 \\
5 \cdot 24 \\
5 \cdot 24\end{array}$ & $\begin{array}{l}5 \cdot 53 \\
5 \cdot 78 \\
5 \cdot 78 \\
5 \cdot 78 \\
5 \cdot 78 \\
5 \cdot 78 \\
5 \cdot 78 \\
5 \cdot 78 \\
5 \cdot 78\end{array}$ & $\begin{array}{c}5 \cdot 14 \\
5 \cdot 25 \\
5 \cdot 33 \\
5 \cdot 38 \\
5 \cdot 43 \\
- \\
- \\
- \\
-\end{array}$ \\
\hline $0 \cdot 75$ & $\begin{array}{l}1 \\
0 \cdot 8 \\
0 \cdot 66 \\
0 \cdot 57 \\
0 \cdot 5 \\
0 \cdot 4 \\
0 \cdot 33 \\
0 \cdot 25 \\
0 \cdot 2\end{array}$ & $\begin{array}{l}4.94 \\
4.98 \\
4.98 \\
4.98 \\
4.98 \\
4.98 \\
4.98 \\
4.98 \\
4.98\end{array}$ & $\begin{array}{l}\mathbf{5} \cdot 32 \\
\mathbf{5 \cdot 3 6} \\
\mathbf{5} \cdot 36 \\
\mathbf{5 \cdot 3 6} \\
\mathbf{5 \cdot 3 6} \\
\mathbf{5 \cdot 3 6} \\
\mathbf{5 \cdot 3 6} \\
\mathbf{5 \cdot 3 6} \\
\mathbf{5 \cdot 3 6}\end{array}$ & $\begin{array}{l}5 \cdot 13 \\
5 \cdot 17 \\
5 \cdot 17 \\
5 \cdot 17 \\
5 \cdot 17 \\
5 \cdot 17 \\
5 \cdot 17 \\
5 \cdot 17 \\
5 \cdot 17\end{array}$ & $\begin{array}{l}\mathbf{5 \cdot 5 3} \\
\mathbf{5 \cdot 5 3} \\
\mathbf{5 \cdot 5 3} \\
\mathbf{5 \cdot 5 3} \\
\mathbf{5 \cdot 5 3} \\
\mathbf{5 \cdot 5 3} \\
\mathbf{5 \cdot 5 3} \\
\mathbf{5 \cdot 5 3} \\
\mathbf{5 \cdot 5 3}\end{array}$ & $\begin{array}{c}5 \cdot 14 \\
5 \cdot 14 \\
5 \cdot 14 \\
5 \cdot 14 \\
5 \cdot 14 \\
- \\
- \\
- \\
-\end{array}$ \\
\hline 1 & $\begin{array}{l}1 \\
0 \cdot 8 \\
0 \cdot 66 \\
0 \cdot 57 \\
0 \cdot 5 \\
0 \cdot 4 \\
0 \cdot 33 \\
0 \cdot 25 \\
0 \cdot 2\end{array}$ & $\begin{array}{l}4.94 \\
4.94 \\
4.94 \\
4.94 \\
4.94 \\
4.94 \\
4.94 \\
4.94 \\
4.94 \\
\end{array}$ & $\begin{array}{l}\mathbf{5} \cdot 32 \\
\mathbf{5} \cdot 30 \\
\mathbf{5} \cdot 30 \\
\mathbf{5} \cdot 30 \\
\mathbf{5} \cdot 30 \\
\mathbf{5 \cdot 3 0} \\
\mathbf{5 \cdot 3 0} \\
\mathbf{5 \cdot 3 0} \\
\mathbf{5 \cdot 3 0}\end{array}$ & $\begin{array}{l}5 \cdot 13 \\
5 \cdot 12 \\
5 \cdot 12 \\
5 \cdot 12 \\
5 \cdot 12 \\
5 \cdot 12 \\
5 \cdot 12 \\
5 \cdot 12 \\
5 \cdot 12\end{array}$ & $\begin{array}{l}\mathbf{5 \cdot 5 3} \\
\mathbf{5 \cdot 5 3} \\
\mathbf{5 \cdot 5 3} \\
\mathbf{5 \cdot 5 3} \\
\mathbf{5 \cdot 5 3} \\
\mathbf{5 \cdot 5 3} \\
\mathbf{5 \cdot 5 3} \\
\mathbf{5 \cdot 5 3} \\
\mathbf{5 \cdot 5 3}\end{array}$ & $\begin{array}{c}5 \cdot 14 \\
5 \cdot 14 \\
5 \cdot 14 \\
5 \cdot 14 \\
5 \cdot 14 \\
- \\
- \\
- \\
-\end{array}$ \\
\hline $1 \cdot 5$ & $\begin{array}{l}1 \\
0 \cdot 8 \\
0 \cdot 66 \\
0 \cdot 57 \\
0 \cdot 5 \\
0 \cdot 4 \\
0 \cdot 33 \\
0 \cdot 25 \\
0 \cdot 2\end{array}$ & $\begin{array}{l}4 \cdot 94 \\
4 \cdot 94 \\
4 \cdot 94 \\
4 \cdot 94 \\
4 \cdot 94 \\
4 \cdot 94 \\
4 \cdot 94 \\
4 \cdot 94 \\
4 \cdot 94\end{array}$ & $\begin{array}{l}\mathbf{5} \cdot 32 \\
\mathbf{5} \cdot \mathbf{3 0} \\
\mathbf{5 \cdot 3 0} \\
\mathbf{5 \cdot 3 0} \\
\mathbf{5} \cdot 30 \\
\mathbf{5} \cdot 30 \\
\mathbf{5} \cdot 30 \\
\mathbf{5 \cdot 3 0} \\
\mathbf{5 \cdot 3 0}\end{array}$ & $\begin{array}{l}5 \cdot 13 \\
5 \cdot 12 \\
5 \cdot 12 \\
5 \cdot 12 \\
5 \cdot 12 \\
5 \cdot 12 \\
5 \cdot 12 \\
5 \cdot 12 \\
5 \cdot 12\end{array}$ & $\begin{array}{l}\mathbf{5 \cdot 5 3} \\
\mathbf{5 \cdot 5 3} \\
\mathbf{5 \cdot 5 3} \\
\mathbf{5 \cdot 5 3} \\
\mathbf{5 \cdot 5 3} \\
\mathbf{5 \cdot 5 3} \\
\mathbf{5 \cdot 5 3} \\
\mathbf{5 \cdot 5 3} \\
\mathbf{5 \cdot 5 3}\end{array}$ & $\begin{array}{c}5 \cdot 14 \\
5 \cdot 14 \\
5 \cdot 14 \\
5 \cdot 14 \\
5 \cdot 14 \\
- \\
- \\
- \\
-\end{array}$ \\
\hline 2 & $\begin{array}{l}1 \\
0 \cdot 8 \\
0 \cdot 66 \\
0 \cdot 57 \\
0 \cdot 5 \\
0 \cdot 4 \\
0 \cdot 33 \\
0 \cdot 25 \\
0 \cdot 2\end{array}$ & $\begin{array}{l}4.94 \\
4 \cdot 94 \\
4 \cdot 94 \\
4.94 \\
4 \cdot 94 \\
4.94 \\
4 \cdot 94 \\
4.94 \\
4 \cdot 94\end{array}$ & $\begin{array}{l}\mathbf{5} \cdot 32 \\
\mathbf{5 \cdot 3 0} \\
\mathbf{5} \cdot 30 \\
\mathbf{5} \cdot 30 \\
\mathbf{5} \cdot 30 \\
\mathbf{5 \cdot 3 0} \\
\mathbf{5 \cdot 3 0} \\
\mathbf{5 \cdot 3 0} \\
\mathbf{5 \cdot 3 0}\end{array}$ & $\begin{array}{l}5 \cdot 13 \\
5 \cdot 12 \\
5 \cdot 12 \\
5 \cdot 12 \\
5 \cdot 12 \\
5 \cdot 12 \\
5 \cdot 12 \\
5 \cdot 12 \\
5 \cdot 12\end{array}$ & $\begin{array}{l}\mathbf{5 \cdot 5 3} \\
\mathbf{5 \cdot 5 3} \\
\mathbf{5 \cdot 5 3} \\
\mathbf{5 \cdot 5 3} \\
\mathbf{5 \cdot 5 3} \\
\mathbf{5 \cdot 5 3} \\
\mathbf{5 \cdot 5 3} \\
\mathbf{5 \cdot 5 3} \\
\mathbf{5 \cdot 5 3}\end{array}$ & $\begin{array}{c}5 \cdot 14 \\
5 \cdot 14 \\
5 \cdot 14 \\
5 \cdot 14 \\
5 \cdot 14 \\
- \\
- \\
- \\
-\end{array}$ \\
\hline
\end{tabular}

Bold signifies cases where the zone of plastic yielding does not penetrate the bottom layer. The exact solution will be $N_{\mathrm{c}}^{*}=5 \cdot 14$, the Prandtl solution.

where

$$
\begin{aligned}
& \theta_{i}=\cos ^{-1}\left(\cos \theta+\frac{H}{r}\right) \\
& n=\frac{c_{\mathrm{u} 2}}{c_{\mathrm{u} 1}}-1
\end{aligned}
$$

and a least upper bound is found by satisfying

$$
\frac{\partial N_{\mathrm{c}}^{*}}{\partial \theta}=0 \quad \frac{\partial N_{\mathrm{c}}^{*}}{\partial r}=0
$$

For a homogeneous soil profile, these two equations can be solved analytically to give a value of $N_{\mathrm{c}}^{*}=5 \cdot 53$. This is approximately $8 \%$ above the exact Prandtl solution of $N_{\mathrm{c}}^{*}=(2+\pi)$.

The ultimate bearing capacity of a footing resting 

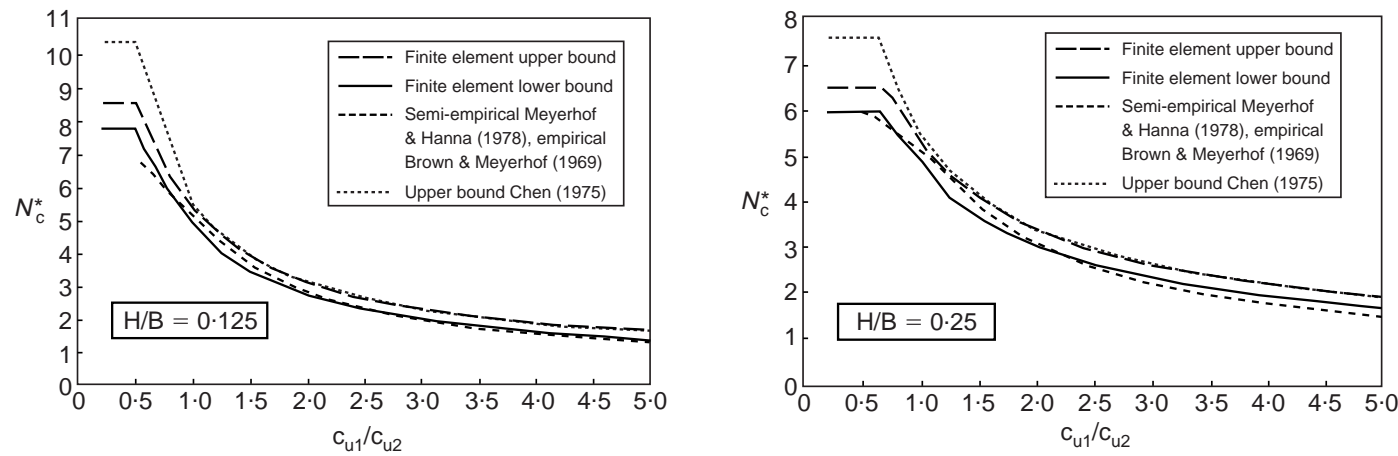

Fig. 7. Variation of bearing capacity factor $N_{\mathrm{c}}^{*}(H / B=0 \cdot 125$ and $H / B=0 \cdot 25)$
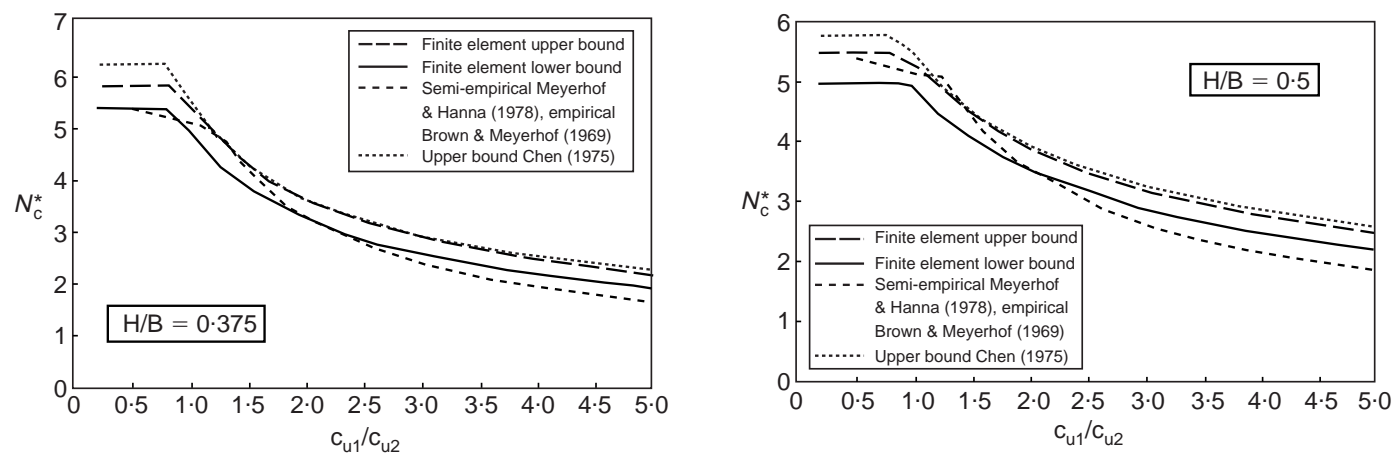

Fig. 8. Variation of bearing capacity factor $N_{\mathrm{c}}^{*}(H / B=0.375$ and $H / B=0.5)$
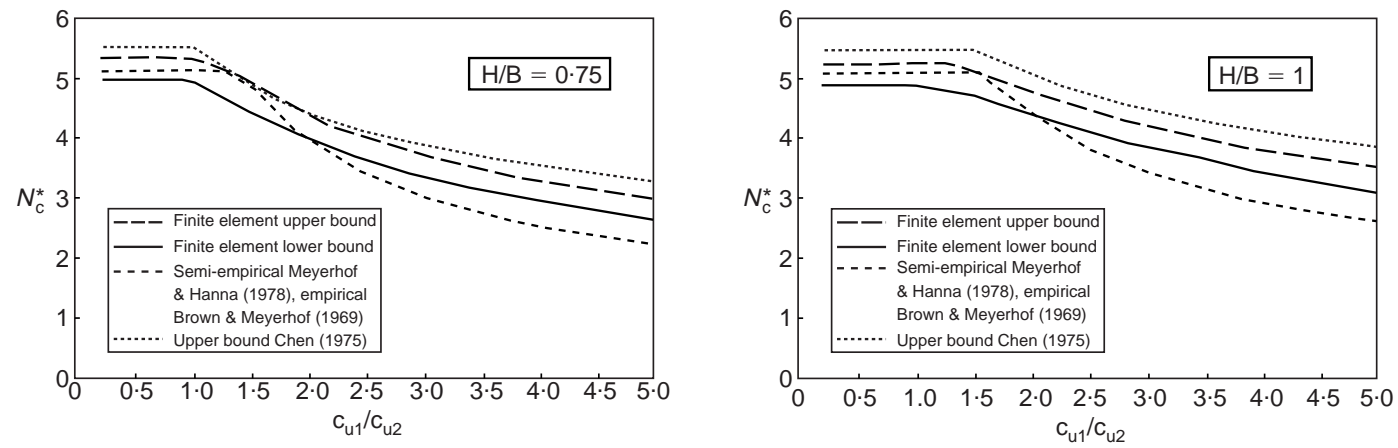

Fig. 9. Variation of bearing capacity factor $N_{\mathrm{c}}^{*}(H / B=0.75$ and $H / B=1)$
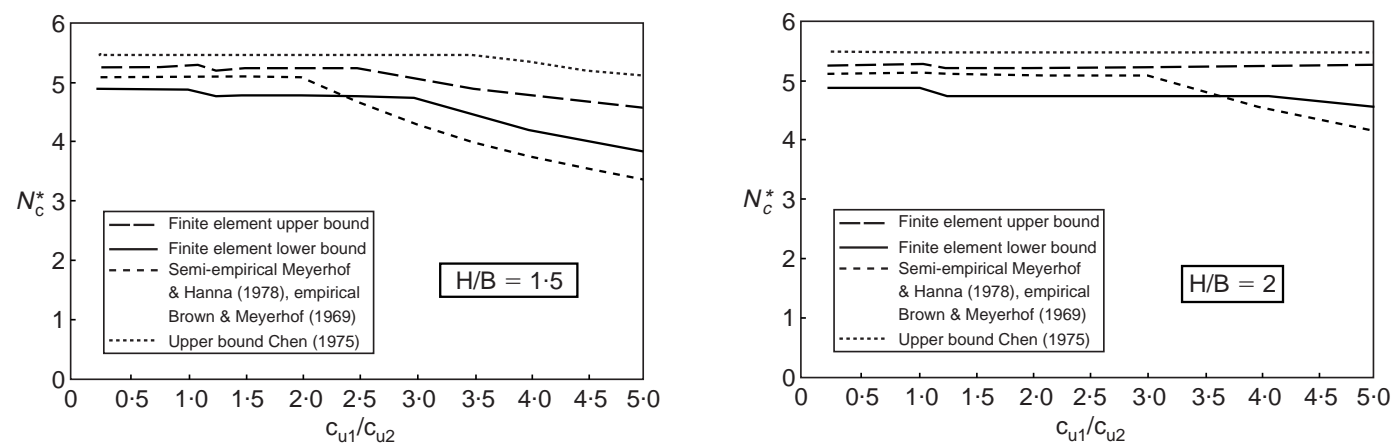

Fig. 10. Variation of bearing capacity factor $N_{c}^{*}(H / B=1.5$ and $H / B=2)$ 
on a strong-over-soft clay deposit, as determined by Meyerhof \& Hanna (1978), is based on the assumption that failure occurs by punching shear through the top layer followed by general shear failure of the bottom layer. The ultimate capacity is given by

$$
q_{\mathrm{u}}=c_{\mathrm{u} 2} N_{\mathrm{c}}+2 c_{\mathrm{a}} H / B
$$

where $N_{\mathrm{c}}=5 \cdot 14$.

In terms of physical behaviour, the second term in this equation is representative of some type of punching shear through the strong top layer, with the first term reflecting full general shear failure in the bottom layer. The term $c_{\mathrm{a}}$ is defined as the unit adhesion acting on the assumed punching shear plane through the strong top crust and was derived from experimental results. The value of $c_{\mathrm{a}}$ varies from unity for a homogeneous profile $\left(c_{\mathrm{u} 1} / c_{\mathrm{u} 2}=1\right)$ to approximately 0.7 for the case of a very strong top layer $\left(c_{\mathrm{u} 1} / c_{\mathrm{u} 2}=10\right)$. This suggests that the full value of punching shear is not developed and was supported by test observations made by Brown \& Meyerhof (1969). Equation (9) can be rearranged to give the bearing capacity factor $N_{\mathrm{c}}^{*}$ as

$$
N_{\mathrm{c}}^{*}=\frac{q_{\mathrm{u}}}{c_{\mathrm{u} 1}}=N_{\mathrm{c}}\left(\frac{c_{\mathrm{u} 2}}{c_{\mathrm{u} 1}}\right)+2\left(\frac{c_{\mathrm{a}}}{c_{\mathrm{u} 1}}\right)\left(\frac{H}{B}\right)
$$

Extensive vertical and

lateral deformation of

upper layer soil column

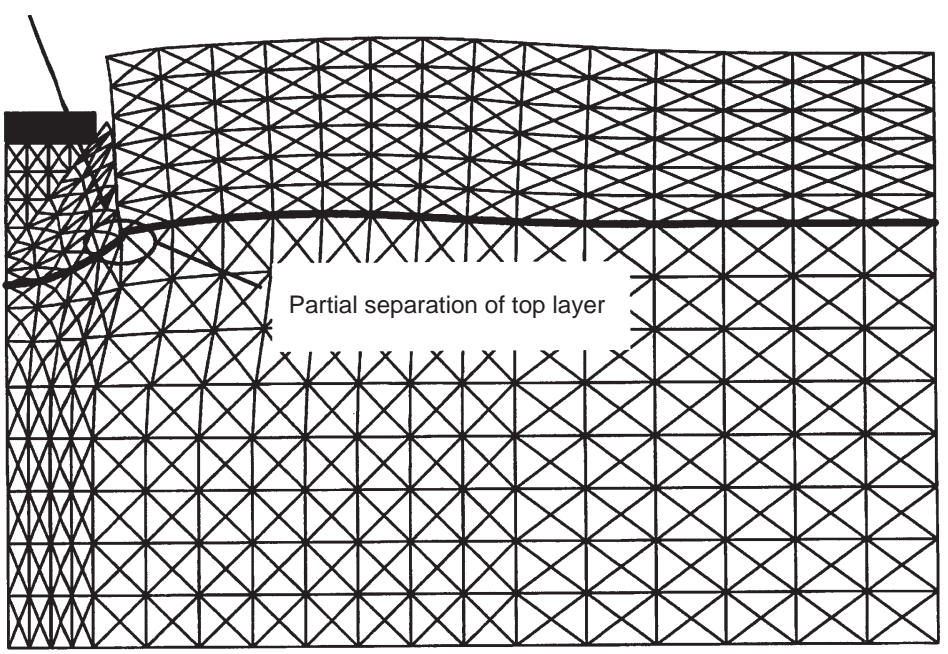

Partial punching shear failure

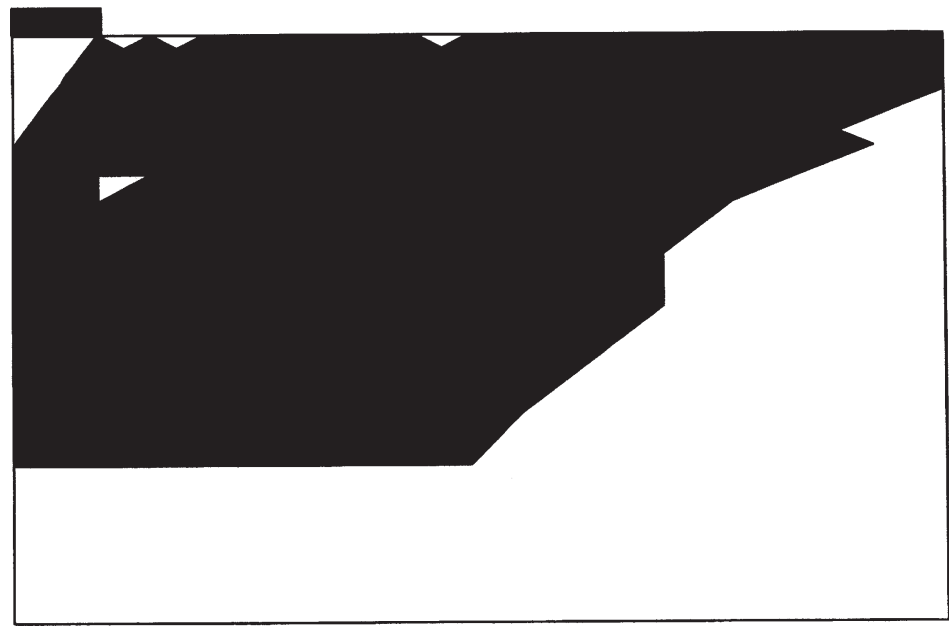

Fig. 11. Deflected mesh and zone of plastic yielding for case of partial punching shear failure $\left(H / B=1, c_{\mathrm{u} 1} / c_{\mathrm{u} 2}=2\right)$ 
Brown \& Meyerhof (1969) provided charts of the bearing capacity factor $N_{\mathrm{c}}^{*}$ for both strong-oversoft and soft-over-strong clay profiles based on a series of model laboratory tests. Their results for the soft-over-strong case are reproduced in Figs 710 for comparison purposes.

\section{Footings on strong clay overlying soft clay}

The upper and lower bound results clearly indicate that a complex relationship exists between general, local and punching shear failure and the ratios $c_{\mathrm{u} 1} / c_{\mathrm{u} 2}$ and $H / B$. Failure generally occurs by either partial or full punching shear through the top layer followed by yielding of the bottom layer. The distinction between these two failure modes is illustrated in Figs 11 and 12. Full punching shear (Fig. 12) is characterized by a complete vertical separation of the top layer, which then effectively acts as a rigid column of soil that punches through to the bottom layer. Conversely, only a small vertical separation of the top layer is evident for partial punching shear (Fig. 11), with both local vertical and lateral deformation of the soil column below the footing now apparent. Full punching shear typically occurs for ratios of $H / B \leqslant 0 \cdot 5$, regardless of the ratio $c_{\mathrm{u} 1} / c_{\mathrm{u} 2}$, while for $H / B>0.5$, the division between full and partial

Minor deformation of

upper layer soil column

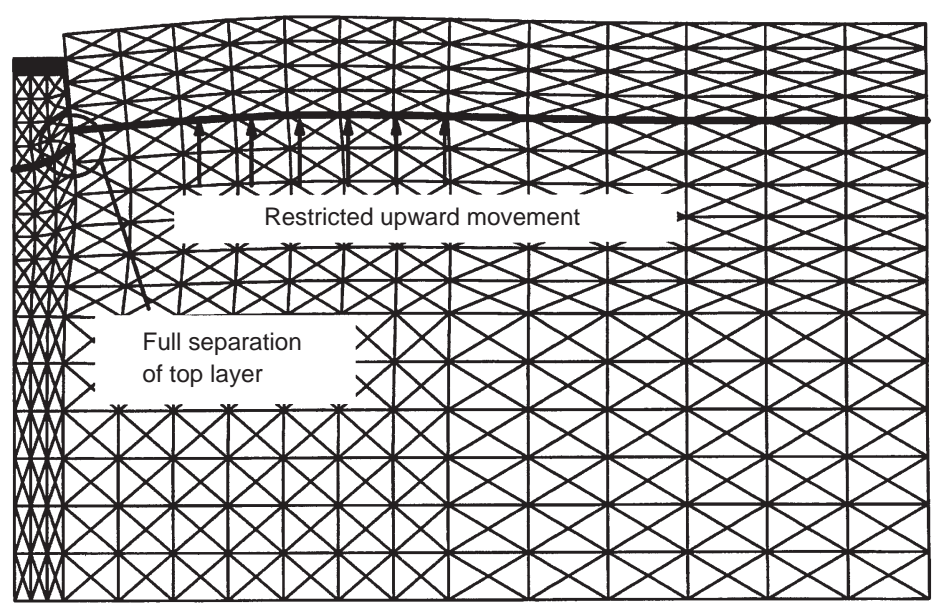

Punching shear failure

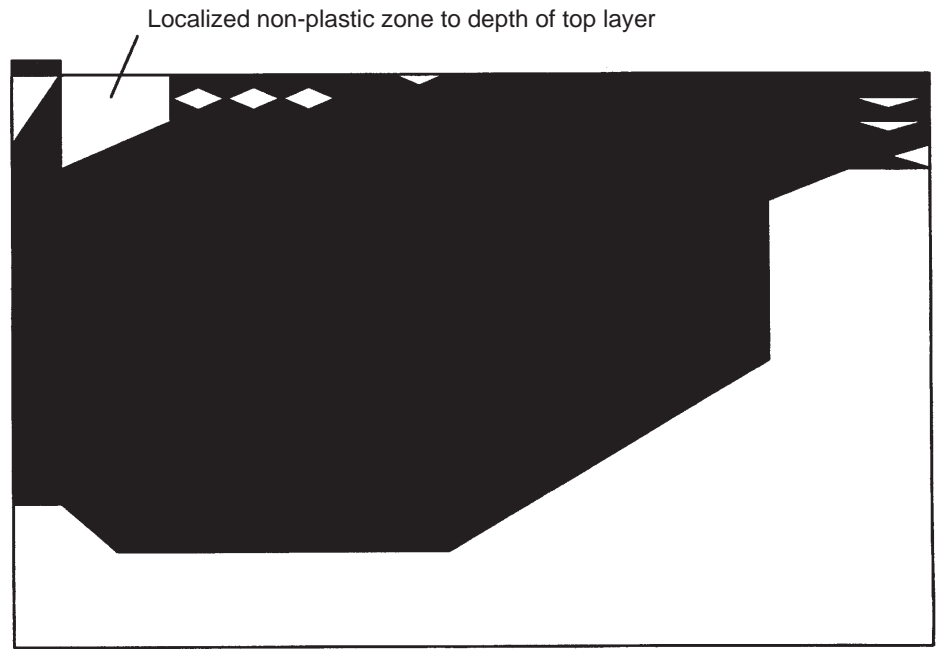

Fig. 12. Deflected mesh and zone of plastic yielding for the case of punching shear failure $\left(H / B=1, c_{\mathrm{u} 1} / c_{\mathrm{u} 2}=5\right)$ 
punching shear occurs at $c_{\mathrm{u} 1} / c_{\mathrm{u} 2}$ approximately equal to $2 \cdot 5$. The extent and form of yielding within the bottom layer is dependent on both the depth and strength of the overlying top layer. This is best illustrated by the velocity diagrams shown in Figs 13-15.

For the case of moderately strong crusts $\left(c_{\mathrm{u} 1} / c_{\mathrm{u} 2} \leqslant 2 \cdot 5\right)$, failure is generally caused by partial punching shear. For thin top crusts with $H / B<0 \cdot 5$, the overall failure mechanism is similar to that depicted in Fig. 6. As the depth of the top crust approaches the footing width $B$, upward deformations within the bottom layer become restricted, causing an increase in the extent of plastic yielding (see Figs 13(a), 14(a) and 15(a)).

As the top crust becomes very strong compared to the bottom layer $\left(c_{\mathrm{u} 1} / c_{\mathrm{u} 2} \geqslant 2 \cdot 5\right)$, full punching shear through the top layer occurs. The very strong top layer then serves to greatly restrict both lateral and vertical movement of the soil contained in the soft layer below (see Figs 13(b), 14(b) and 15(b)). This results in the formation of a deep zone of plastic shearing within the bottom layer and, for thicker crusts $(H / B>0 \cdot 75)$, a local elastic zone is formed within the top layer immediately adjacent to the footing as shown in Fig. 12.

The limit analysis results indicate that a reduction in bearing capacity for a strong-over-soft clay system occurs up to a depth ratio of $H / B \approx$ $1 \cdot 5-2 \cdot 0$. This lower limit is applicable for soil profiles where $c_{\mathrm{u} 1} / c_{\mathrm{u} 2} \leqslant 2 \cdot 5$, but for profiles that have a very strong top crust with $c_{\mathrm{u} 1} / c_{\mathrm{u} 2} \geqslant 2 \cdot 5$, punching failure through the top layer is likely to occur up to a depth ratio of $H / B=2$. For ratios of $H / B>2$, failure is contained entirely within the top layer and is independent of the ratio $c_{\mathrm{u} 1} / c_{\mathrm{u} 2}$. These results are similar to those predicted by Chen (1975), but are lower than those estimated by Meyerhof \& Hanna (1978), who suggest that a reduction in bearing capacity may occur up to a depth ratio of $H / B \simeq 2 \cdot 5$.

The analytical upper bounds obtained by Chen (1975), who assumed a simple circular failure mechanism, compare favourably with those ob-

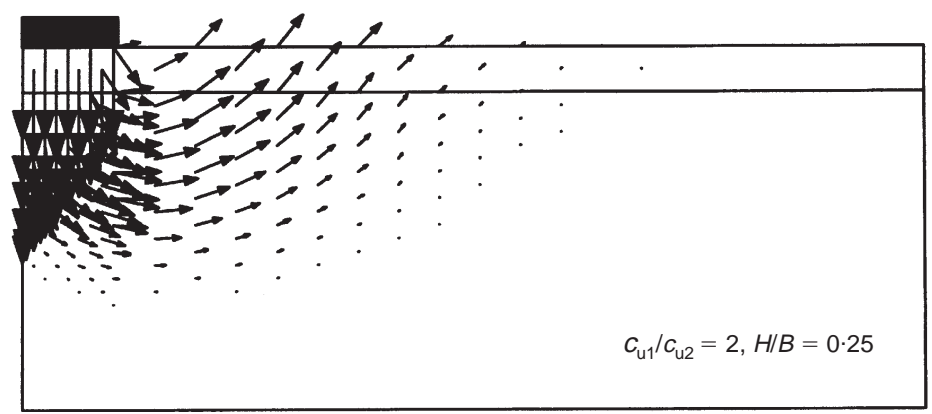

(a)

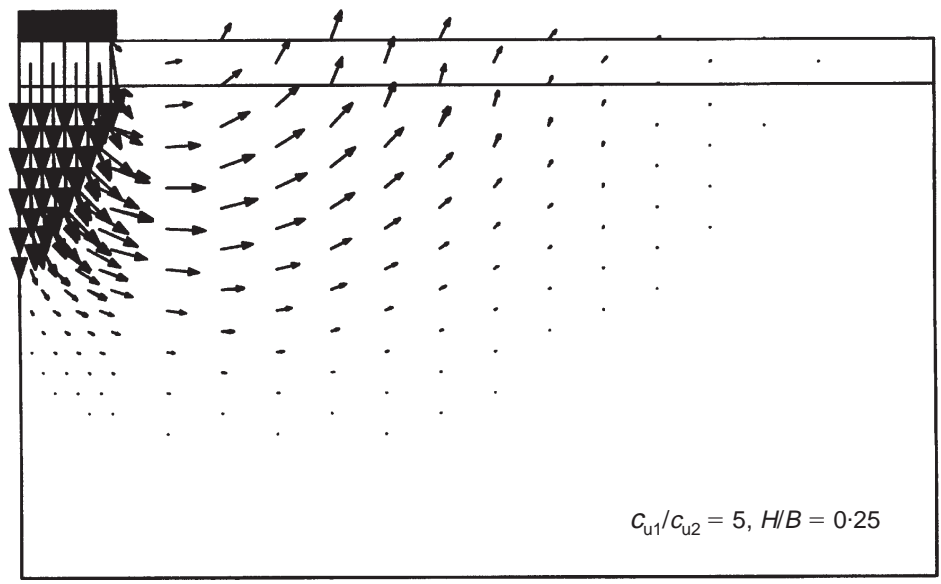

(b)

Fig. 13. Velocity diagrams for strong-over-soft layers $\left(c_{\mathrm{u} 1} / c_{\mathrm{u} 2}=2,5\right.$ and $\boldsymbol{H} / \boldsymbol{B}=\mathbf{0} \cdot \mathbf{2 5})$ 


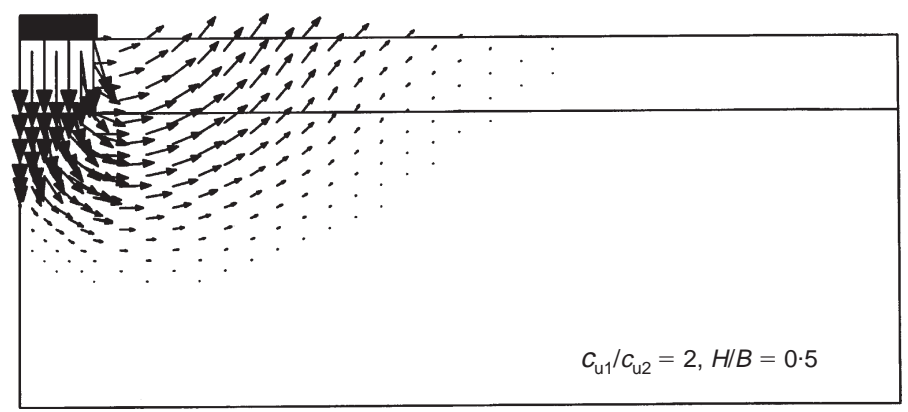

(a)

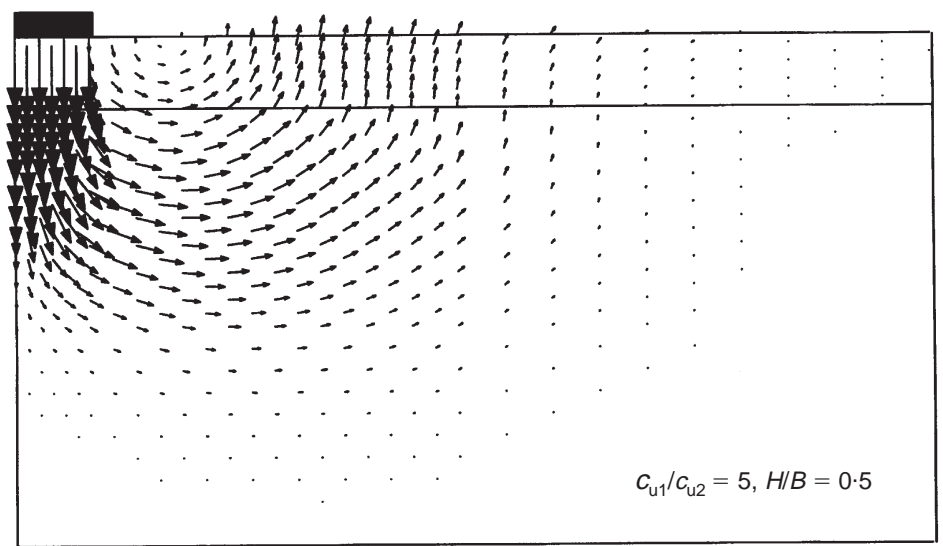

(b)

Fig. 14. Velocity diagrams for strong-over-soft layers $\left(c_{\mathrm{u} 1} / c_{\mathrm{u} 2}=2,5\right.$ and $\boldsymbol{H} / \boldsymbol{B}=\mathbf{0 . 5})$

tained from the finite element upper bounds for smaller values of $H / B$ but become rather unconservative when $H \geqslant B$.

For ratios of $H / B \leqslant 0 \cdot 5$, the solutions of Chen are less than $5 \%$ above the upper bound limit analysis results for all values of $c_{\mathrm{u} 1} / c_{\mathrm{u} 2}$ (Figs 7 and 8). For larger values of $H / B$, the solutions of Chen become increasingly inaccurate as $c_{\mathrm{u} 1} / c_{\mathrm{u} 2}$ increases, with a maximum error of approximately $15 \%$ for $H / B=1.5$ and $c_{\mathrm{u} 1} / c_{\mathrm{u} 2}=5$. The reason for this is that for larger values of $H / B$ and $c_{\mathrm{u} 1} / c_{\mathrm{u} 2}$, the assumed mechanism of Chen (1975) is no longer a good representation of the true collapse mechanism. This is illustrated in Fig. 16, where the optimal mechanism using a circular failure surface does not penetrate deeply into the weak layer. The finite element limit analysis results clearly indicate that the failure mechanism that yields the best upper bound penetrates deeply into the soft bottom layer.

With reference to Figs $7-10$, it can be seen that for a soil profile having a moderately strong top crust $\left(c_{\mathrm{u} 1} / c_{\mathrm{u} 2} \leqslant 2 \cdot 5\right)$, the solutions of Meyerhof \&
Hanna (1978) typically lie either within or just outside the upper and lower bound solutions. For very strong top crusts $\left(c_{\mathrm{u} 1} / c_{\mathrm{u} 2}>2 \cdot 5\right)$, these solutions tend to become overconservative as $H / B$ increases, and lie $12-16 \%$ below the lower bound solution.

As with the solutions of Chen (1975), the solutions of Meyerhof \& Hanna (1978) are limited by their assumption that a single type of failure mechanism exists. Only for the restricted case of thin, moderately strong crusts, where $H / B \leqslant 0.5$ and $c_{\mathrm{u} 1} / c_{\mathrm{u} 2} \leqslant 2.5$ (Figs 13(a) and 14(a)), does the assumption of punching through the crust followed by general shear failure in the bottom layer appear, to some degree, to be valid. This assumption is clearly not correct for larger top crust thicknesses (Fig. 15(a)) or if the crust is substantially stronger than the bottom layer (Figs 13(b), 14(b) and 15(b)). For these cases, failure tends to be either a combination of general shear failure through both layers or a deep rotational mechanism, depending on the ratio of the layer strengths $c_{\mathrm{u} 1} / c_{\mathrm{u} 2}$. 


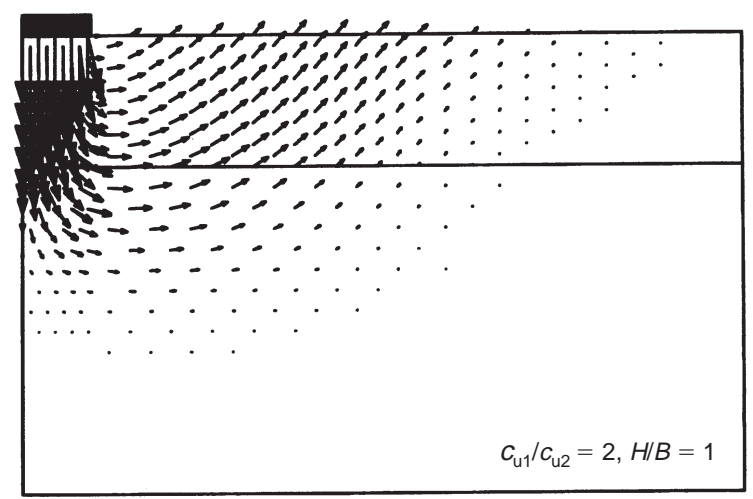

(a)

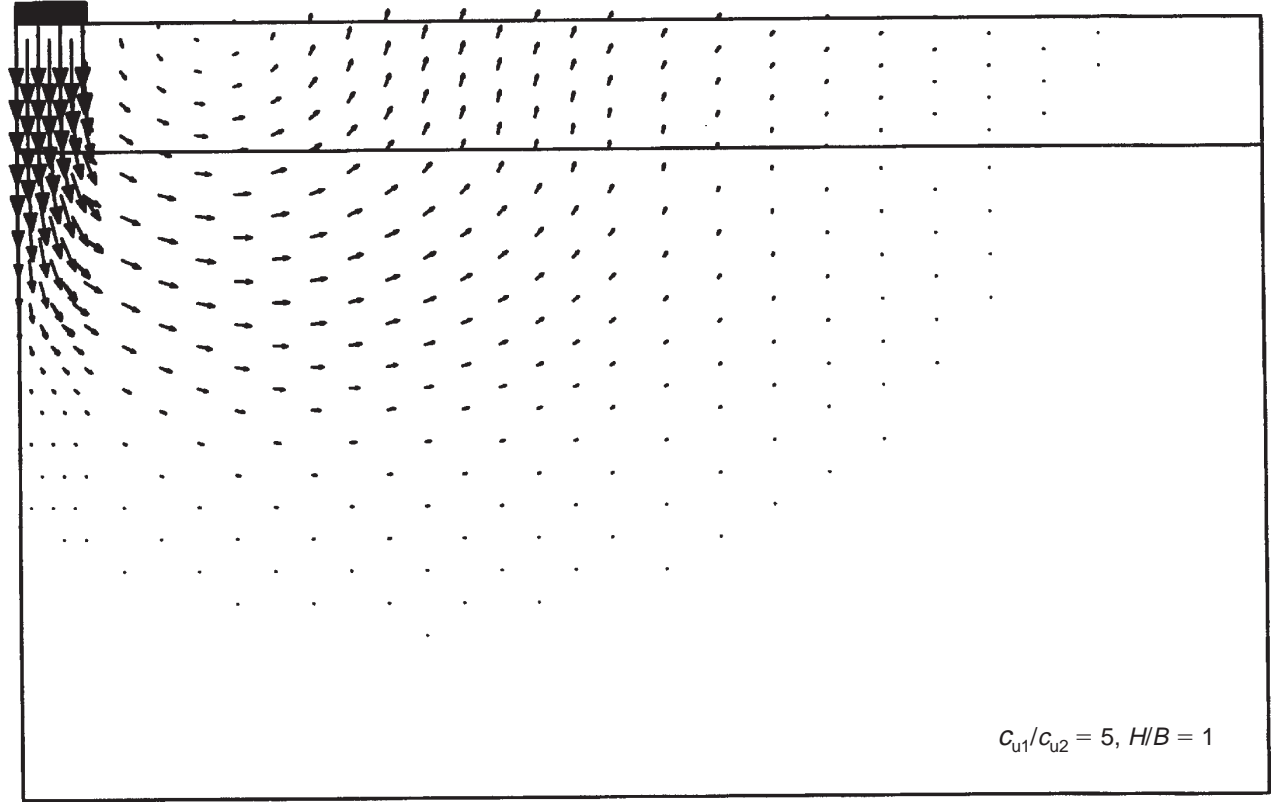

(b)

Fig. 15. Velocity diagrams for strong-over-soft layers $\left(c_{\mathrm{u} 1} / c_{\mathrm{u} 2}=2,5\right.$ and $\left.H / B=1\right)$

Footings on soft clay overlying strong clay

The upper and lower bound results indicate that for ratios of $H / B \leqslant 0 \cdot 5$, the bearing capacity increases as the relative strength of the bottom layer rises. For all of these cases, the proportion of yielding within the bottom layer decreases as its strength increases. At a limiting ratio of $c_{\mathrm{u} 1} / c_{\mathrm{u} 2}$, no further increase in bearing capacity is achieved as the failure surface becomes fully contained within the top layer. This is illustrated in Figs 17 and 18 , and is represented by the sudden change in the curvature of the plots shown in Figs 7-10. As an example, for $H / B=0 \cdot 125$ (Fig. 7), the bearing capacity increases as $c_{\mathrm{u} 1} / c_{\mathrm{u} 2}$ decreases until a limiting value of $c_{\mathrm{u} 1} / c_{\mathrm{u} 2}=0.5$ is reached. After this point, failure is fully contained within the top layer, as shown in Fig. 17(c).

For all values of $H / B>0 \cdot 5$, the bound solutions indicate that failure occurs entirely within the top layer and the bearing capacity is independent of the strength of the bottom layer.

The upper bound solutions of Chen (1975) overestimate the bearing capacity factor for all cases where $c_{\mathrm{u} 1} / c_{\mathrm{u} 2}<1$ and are $5-22 \%$ higher than the finite element upper bound solutions. The overestimate is greatest for small top layer thicknesses where $H / B \leqslant 0 \cdot 375$. The reason for this is illustrated in Fig. 17(b), where it can be seen that the 


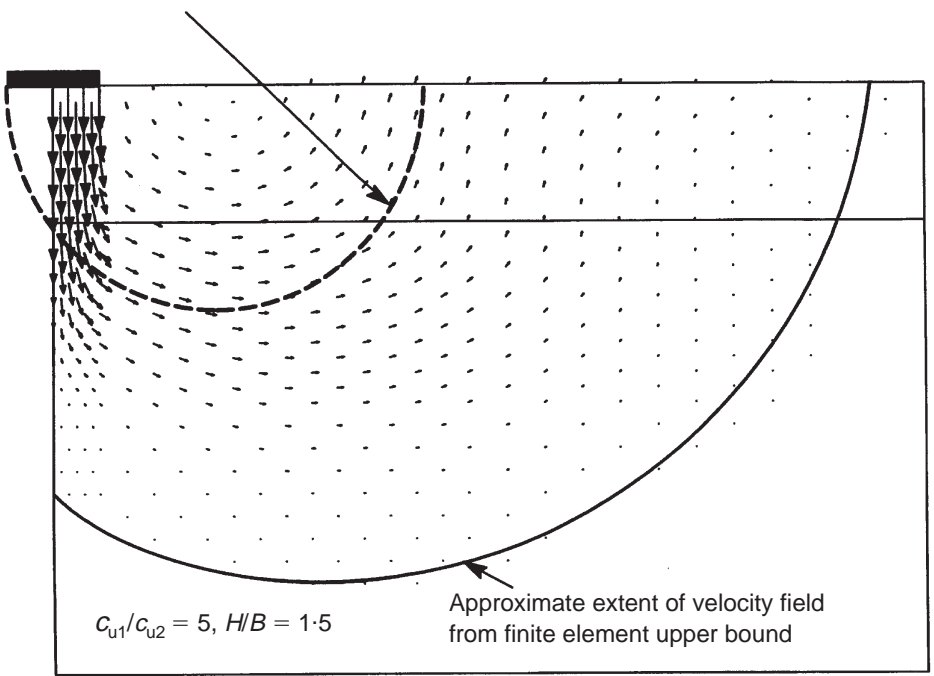

Fig. 16. Comparison of velocity diagrams for strong-over-soft layers $\left(c_{\mathrm{u} 1} / c_{\mathrm{u} 2}=5\right.$ and $\left.H / B=1 \cdot 5\right)$

optimal slip circle determined by Chen (1975) penetrates deeper into the underlying strong layer than the mechanism predicted by the finite element solution. When the failure mechanism is contained within the thin top layer, Figs 17(c) and 18(c) suggest that failure is by lateral squeezing and local failure at the footing edge and is therefore not accurately modelled by a circular slip mechanism.

As $H / B$ increases above $0 \cdot 375$, the accuracy of the Chen (1975) solutions improves and typically lies $3-7 \%$ above those of the finite element upper bound solutions. This is because the majority of yielding occurs within the top layer and the actual failure mode can now be adequately modelled by a rotational failure mechanism. For $H / B \geqslant 0.75$, failure occurs entirely within the top layer and the exact solution for these cases (in bold in Table 2) will be $N_{\mathrm{c}}^{*}=5 \cdot 14$, the Prandtl solution. This implies that the error in the finite element upper bound solutions is $\approx 3 \%$, while the error in the Chen (1975) solutions is $\approx 6-7 \%$.

The empirical results given by Brown \& Meyerhof (1969) are limited to $c_{\mathrm{u} 1} / c_{\mathrm{u} 2}$ ratios between 1 and 0.5 and are given in Table 2. For relatively thin top layers with $H / B \leqslant 0 \cdot 5$, the solutions of Brown \& Meyerhof (1969) typically lie near the finite element lower bound solutions. As the top layer thickness increases above $H / B>0.5$, failure becomes contained within it and the Brown \& Meyerhof (1969) solution lies central to both upper and lower bound finite element solutions.

\section{Effect of footing roughness}

The upper and lower bound limit analysis results presented so far have been for perfectly rough footings. The effect of soil-footing interface strength on the ultimate bearing capacity has been determined by modelling a perfectly smooth footing, with the results shown in Table 3.

For a strong-over-soft clay profile, the soil-footing interface strength has little $(<2 \%)$ or no effect on the calculated bearing capacity. The results given in Table 1 can therefore be used to determine the bearing capacity of both perfectly rough and smooth surface footings. Similarly, for a softover-strong clay system where $H / B>0.5$, the bearing capacity does not vary with footing roughness and the results given in Table 2 are appropriate for perfectly rough and smooth footings.

For a soft-over-strong clay system where $H / B \leqslant 0.5$, a perfectly smooth soil-footing interface serves to reduce the bearing capacity by up to $25 \%$ for $H / B=0 \cdot 125$. This difference reduces to around $3 \%$ for $H / B=0 \cdot 5$. Results are shown in Table 3.

\section{CONCLUSIONS}

The undrained bearing capacity of a surface strip footing resting on a layered clay profile has been investigated. Using recent numerical formulations of the upper and lower bound limit theorems, rigorous bounds on the bearing capacity for a wide range of problem geometries have been obtained, 


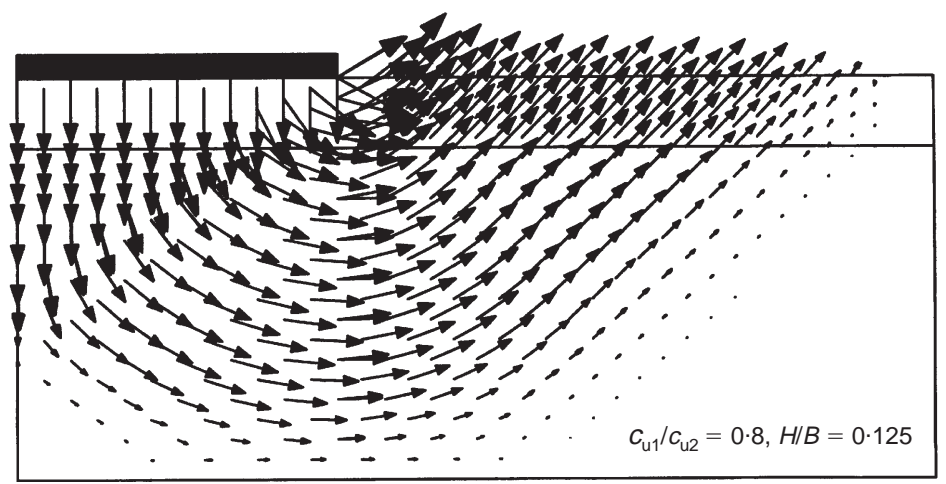

(a)
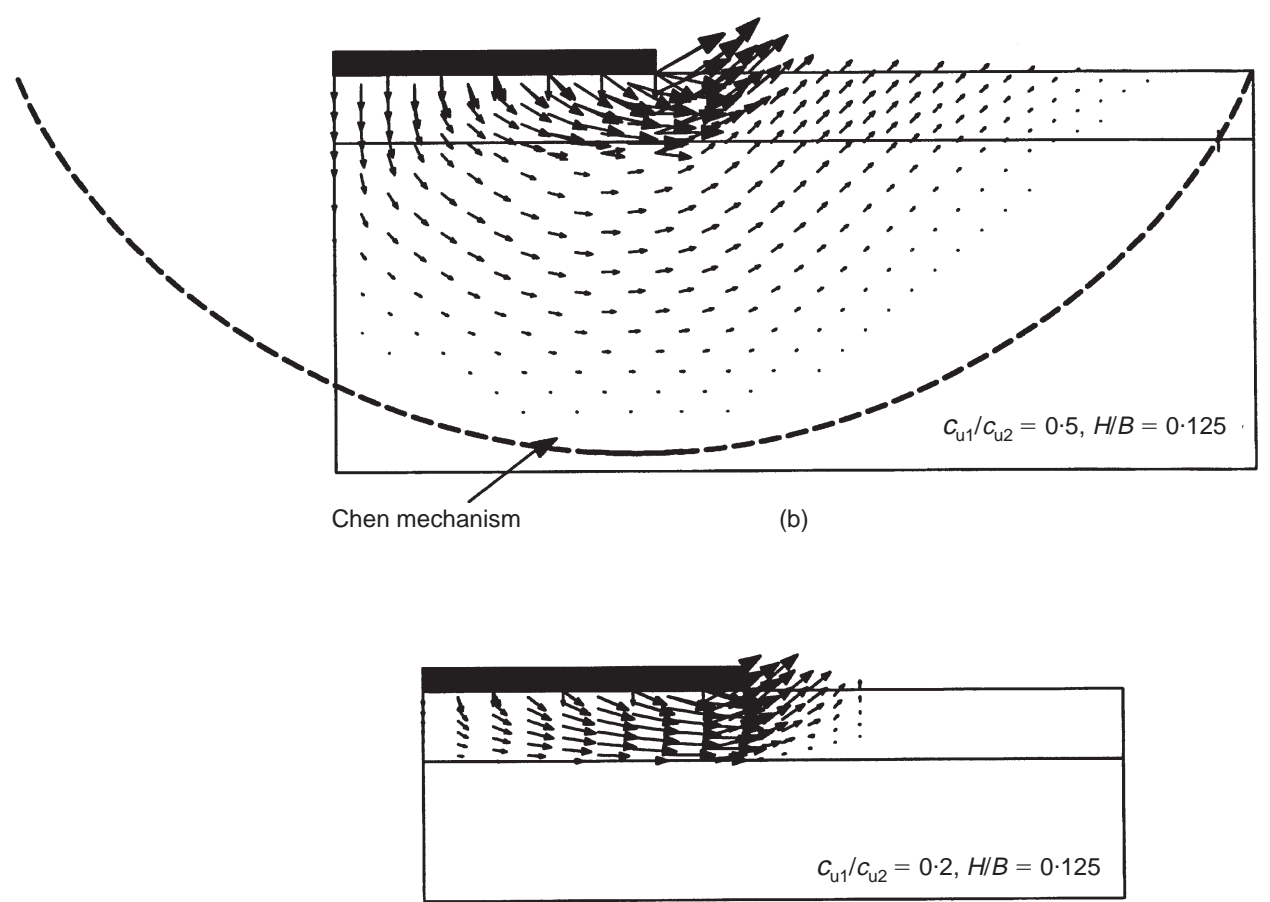

(c)

Fig. 17. Velocity diagrams for soft-over-strong layers $\left(c_{\mathrm{u} 1} / c_{\mathrm{u} 2}=0 \cdot 8,0 \cdot 5,0 \cdot 2\right.$ and $\left.H / B=0 \cdot 125\right)$

with the exact collapse load typically being bracketed to within $12 \%$. The results obtained have been presented in terms of a modified bearing capacity factor $N_{\mathrm{c}}^{*}$ in both graphical and tabular form to facilitate their use in solving practical design problems.

The following conclusions can be made based on the limit analysis results:

(a) For a strong-over-soft clay profile, a number of different failure mechanisms exist that are functions of both the crust thickness and its strength relative to the underlying weaker layer. For this reason, existing upper bound and semi-empirical solutions that are based on a single assumed failure surface are unable to model the likely failure mode over a large range of problem geometries.

(b) Existing upper bound, empirical and semiempirical solutions can differ from the bound solutions by up to $\pm 20 \%$. The existing solutions are in greatest error when the top 


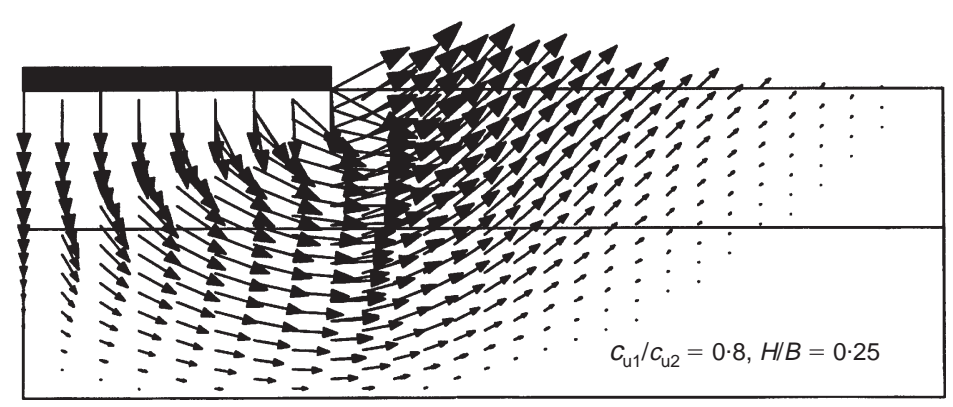

(a)

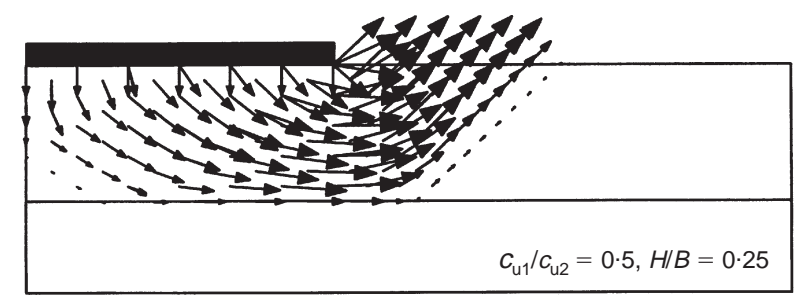

(b)

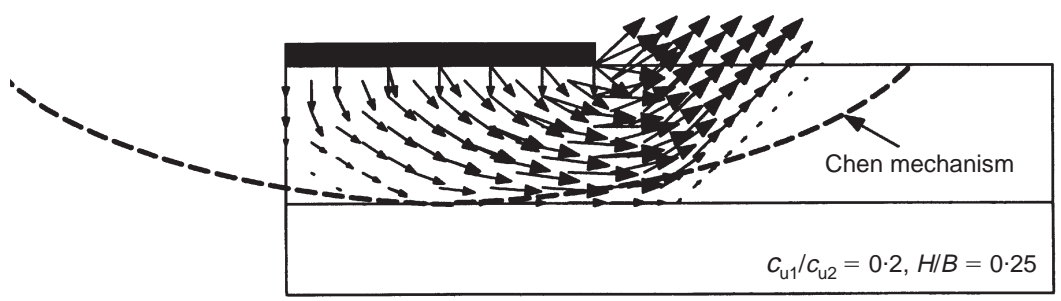

(c)

Fig. 18. Velocity diagrams for soft-over-strong layers $\left(c_{\mathrm{u} 1} / c_{\mathrm{u} 2}=0 \cdot 8,0 \cdot 5,0 \cdot 2\right.$ and $\boldsymbol{H} / \boldsymbol{B}=\mathbf{0} \cdot \mathbf{2 5})$

layer is very strong compared to the bottom layer $\left(c_{\mathrm{u} 1} / c_{\mathrm{u} 2}>2.5\right)$ and/or its depth is greater than half the footing width $(H / B>0 \cdot 5)$.

(c) A reduction in bearing capacity for a strongover-soft clay system occurs up to a depth ratio of $H / B \approx 1 \cdot 5-2 \cdot 0$, where the lower limit is applicable for soil profiles with $c_{\mathrm{u} 1} / c_{\mathrm{u} 2} \leqslant 2 \cdot 5$. For depth ratios of $H / B>2$, failure is likely to be fully contained within the top layer, and the bearing capacity is given by the Prandtl solution $N_{\mathrm{c}}^{*}=2+\pi$.

(d) For a soft-over-strong clay system where $H / B \leqslant 0 \cdot 5$, the bearing capacity is likely to increase as the relative strength of the bottom layer rises. For thicker top layers where $H / B>0.5$, failure occurs entirely within the top layer and the bearing capacity is given by the Prandtl solution $N_{\mathrm{c}}^{*}=2+\pi$.

(e) For a soft-over-strong clay system where
$H / B \leqslant 0 \cdot 5$, the effect of footing roughness is important and can lead to a reduction in bearing capacity by as much as $25 \%$. For a soft-over-strong clay system where $H / B>0.5$, and for strong-over-soft soil profiles, the bearing capacity is not affected by footing roughness.

\section{REFERENCES}

Anderheggen, E. \& Knöpfel, H. (1972). Finite element limit analysis using linear programming. Int. J. Solids Struct. 8, 1413-1431.

Bottero, A., Negre, R., Pastor, J. \& Turgeman, S. (1980). Finite element method and limit analysis theory for soil mechanics problems. Comput. Methods Appl. Mech. Engng 22, 131-149.

Brown, J. D. \& Meyerhof, G. G. (1969). Experimental study of bearing capacity in layered clays. Proc. 7th Int. Conf. Soil Mech. Found. Engng, Mexico 2, 45-51. 
Table 3. Values of bearing capacity factor $N_{\mathrm{c}}^{*}$ for smooth footings $c_{\mathrm{u} 1} / c_{\mathrm{u} 2} \leqslant 1$

\begin{tabular}{|c|c|c|c|c|c|c|}
\hline \multirow[t]{2}{*}{$H / B$} & \multirow[t]{2}{*}{$c_{\mathrm{u} 1} / c_{\mathrm{u} 2}$} & \multicolumn{5}{|c|}{ Values of bearing capacity factor $N_{\mathrm{c}}^{*}$} \\
\hline & & $\begin{array}{l}\text { Lower bound } \\
\text { (a) }\end{array}$ & $\begin{array}{l}\text { Upper bound } \\
\text { (b) }\end{array}$ & $\begin{array}{c}\text { Average } \\
((\mathrm{a})+(\mathrm{b})) / 2\end{array}$ & $\begin{array}{l}\text { Upper bound } \\
\text { (Chen, 1975) }\end{array}$ & $\begin{array}{c}\text { Brown \& } \\
\text { Meyerhof (1969) }\end{array}$ \\
\hline $0 \cdot 125$ & $\begin{array}{l}1 \\
0 \cdot 8 \\
0 \cdot 66 \\
0 \cdot 57 \\
0 \cdot 5 \\
0 \cdot 4 \\
0 \cdot 33 \\
0 \cdot 25 \\
0 \cdot 2\end{array}$ & $\begin{array}{l}4 \cdot 86 \\
5 \cdot 58 \\
5 \cdot 88 \\
5 \cdot 94 \\
5 \cdot 94 \\
5 \cdot 94 \\
5 \cdot 94 \\
5 \cdot 94 \\
5 \cdot 94\end{array}$ & $\begin{array}{l}5 \cdot 32 \\
6 \cdot 06 \\
6 \cdot 50 \\
6 \cdot 50 \\
6 \cdot 50 \\
6 \cdot 50 \\
6 \cdot 50 \\
6 \cdot 50 \\
6 \cdot 50\end{array}$ & $\begin{array}{l}5 \cdot 09 \\
5 \cdot 82 \\
6 \cdot 19 \\
6 \cdot 22 \\
6 \cdot 22 \\
6 \cdot 22 \\
6 \cdot 22 \\
6 \cdot 22 \\
6 \cdot 22\end{array}$ & $\begin{array}{r}5 \cdot 53 \\
7 \cdot 48 \\
8 \cdot 78 \\
9 \cdot 70 \\
10 \cdot 40 \\
10 \cdot 40 \\
10 \cdot 40 \\
10 \cdot 40 \\
10 \cdot 40\end{array}$ & $\begin{array}{c}5 \cdot 14 \\
5 \cdot 81 \\
6 \cdot 38 \\
6 \cdot 71 \\
6 \cdot 91 \\
- \\
- \\
- \\
-\end{array}$ \\
\hline $0 \cdot 25$ & $\begin{array}{l}1 \\
0 \cdot 8 \\
0 \cdot 66 \\
0 \cdot 57 \\
0 \cdot 5 \\
0 \cdot 4 \\
0 \cdot 33 \\
0 \cdot 25 \\
0 \cdot 2\end{array}$ & $\begin{array}{l}4 \cdot 86 \\
5 \cdot 11 \\
5 \cdot 11 \\
5 \cdot 11 \\
5 \cdot 11 \\
5 \cdot 11 \\
5 \cdot 11 \\
5 \cdot 11 \\
5 \cdot 11\end{array}$ & $\begin{array}{l}5 \cdot 32 \\
5 \cdot 48 \\
5 \cdot 48 \\
5 \cdot 49 \\
5 \cdot 49 \\
5 \cdot 49 \\
5 \cdot 49 \\
5 \cdot 49 \\
5 \cdot 49\end{array}$ & $\begin{array}{l}5 \cdot 09 \\
5 \cdot 30 \\
5 \cdot 30 \\
5 \cdot 30 \\
5 \cdot 30 \\
5 \cdot 30 \\
5 \cdot 30 \\
5 \cdot 30 \\
5 \cdot 30\end{array}$ & $\begin{array}{l}5 \cdot 53 \\
6 \cdot 57 \\
7 \cdot 61 \\
7 \cdot 61 \\
7 \cdot 61 \\
7 \cdot 61 \\
7 \cdot 61 \\
7 \cdot 61 \\
7 \cdot 61\end{array}$ & $\begin{array}{c}5 \cdot 14 \\
5 \cdot 52 \\
5 \cdot 81 \\
5 \cdot 91 \\
6 \cdot 00 \\
- \\
- \\
- \\
-\end{array}$ \\
\hline 0.375 & $\begin{array}{l}1 \\
0 \cdot 8 \\
0 \cdot 66 \\
0 \cdot 57 \\
0 \cdot 5 \\
0 \cdot 4 \\
0 \cdot 33 \\
0 \cdot 25 \\
0 \cdot 2\end{array}$ & $\begin{array}{l}4 \cdot 86 \\
5 \cdot 00 \\
5 \cdot 00 \\
5 \cdot 00 \\
5 \cdot 00 \\
5 \cdot 00 \\
5 \cdot 00 \\
5 \cdot 00 \\
5 \cdot 00\end{array}$ & $\begin{array}{l}\mathbf{5} \cdot 32 \\
\mathbf{5} \cdot 31 \\
\mathbf{5} \cdot 32 \\
\mathbf{5} \cdot 32 \\
\mathbf{5} \cdot 32 \\
\mathbf{5 \cdot 3 2} \\
\mathbf{5 \cdot 3 2} \\
\mathbf{5 \cdot 3 2} \\
\mathbf{5 \cdot 3 2}\end{array}$ & $\begin{array}{l}5 \cdot 09 \\
5 \cdot 16 \\
5 \cdot 16 \\
5 \cdot 16 \\
5 \cdot 16 \\
5 \cdot 16 \\
5 \cdot 16 \\
5 \cdot 16 \\
5 \cdot 16\end{array}$ & $\begin{array}{l}5 \cdot 53 \\
6 \cdot 24 \\
6 \cdot 24 \\
6 \cdot 24 \\
6 \cdot 24 \\
6 \cdot 24 \\
6 \cdot 24 \\
6 \cdot 24 \\
6 \cdot 24\end{array}$ & $\begin{array}{c}5 \cdot 14 \\
5 \cdot 25 \\
5 \cdot 38 \\
5 \cdot 43 \\
5 \cdot 48 \\
- \\
- \\
- \\
-\end{array}$ \\
\hline $0 \cdot 5$ & $\begin{array}{l}1 \\
0 \cdot 8 \\
0 \cdot 66 \\
0 \cdot 57 \\
0 \cdot 5 \\
0 \cdot 4 \\
0 \cdot 33 \\
0 \cdot 25 \\
0 \cdot 2\end{array}$ & $\begin{array}{l}4 \cdot 86 \\
4 \cdot 86 \\
4 \cdot 86 \\
4 \cdot 86 \\
4 \cdot 86 \\
4 \cdot 86 \\
4 \cdot 86 \\
4 \cdot 86 \\
4 \cdot 86\end{array}$ & $\begin{array}{l}5 \cdot 32 \\
5 \cdot 31 \\
5 \cdot 31 \\
5 \cdot 31 \\
5 \cdot 31 \\
5 \cdot 31 \\
5 \cdot 31 \\
5 \cdot 31 \\
5 \cdot 31\end{array}$ & $\begin{array}{l}5 \cdot 09 \\
5 \cdot 09 \\
5 \cdot 09 \\
5 \cdot 09 \\
5 \cdot 09 \\
5 \cdot 09 \\
5 \cdot 09 \\
5 \cdot 09 \\
5 \cdot 09\end{array}$ & $\begin{array}{l}5 \cdot 53 \\
5 \cdot 78 \\
5 \cdot 78 \\
5 \cdot 78 \\
5 \cdot 78 \\
5 \cdot 78 \\
5 \cdot 78 \\
5 \cdot 78 \\
5 \cdot 78\end{array}$ & $\begin{array}{c}5 \cdot 14 \\
5 \cdot 25 \\
5 \cdot 33 \\
5 \cdot 38 \\
5 \cdot 43 \\
- \\
- \\
- \\
-\end{array}$ \\
\hline
\end{tabular}

Bold signifies cases where the zone of plastic yielding does not penetrate the bottom layer. The exact solution will be $N_{\mathrm{c}}^{*}=5 \cdot 14$, the Prandtl solution.

Button, S. J. (1953). The bearing capacity of footings on a two-layer cohesive subsoil. Proc. 3rd Int. Conf. Soil Mech. Found. Engng, Zurich 1, 332-335.

Chen, W. F. (1975). Limit analysis and soil plasticity. Amsterdam: Elsevier.

Davis, E. H. \& Booker, J. R. (1973). The effect of increasing strength with depth on the bearing capacity of clays. Géotechnique 23(4), 551-563.

Florkiewicz, A. (1989). Upper bound to bearing capacity of layered soils. Can. Geotech. J. 26, 730-736.

Lysmer, J. (1970). Limit analysis of plane problems in soil mechanics. J. Soil Mech. Found. Div. ASCE 96, SM4, 1131-1334.

Meyerhof, G. G. \& Hanna, A. M. (1978). Ultimate bearing capacity of foundations on layered soils under inclined load. Can. Geotech. J. 15, 565-572.

Nagtegaal, J. C., Parks, D. M. \& Rice, J. R. (1974). On numerically accurate finite element solutions in the fully plastic range. Computer Methods Appl. Mech. Engng 4, 153-177.

Reddy, A. S. \& Srinivasan, R. J. (1967). Bearing capacity of footings on layered clays. J. Soil Mech. Found. Div., ASCE 93, SM2, 83-99.

Sloan, S. W. (1988). Lower bound limit analysis using finite elements and linear programming. Int. J. Numer. Anal. Methods Geomech. 12, 61-67.

Sloan, S. W. \& Kleeman, P. W. (1995). Upper bound limit analysis using discontinuous velocity fields. Computer Methods Appl. Mech. Engng 127, 293-314.

Sloan, S. W. \& Randolph, M. F. (1982). Numerical prediction of collapse loads using finite element methods. Int. J. Numer. Anal. Methods Geomech. 6, 47-76.

Terzaghi, K. (1943). Theoretical soil mechanics. New York: Wiley. 